PAPER • OPEN ACCESS

Algebro-geometric integration of the Q1 lattice equation via nonlinear integrable symplectic maps

To cite this article: Xiaoxue Xu et al 2021 Nonlinearity 342897

View the article online for updates and enhancements. 


\title{
Algebro-geometric integration of the Q1 lattice equation via nonlinear integrable symplectic maps
}

\author{
Xiaoxue $\mathrm{Xu}^{1}$, Cewen $\mathrm{Cao}^{1}$ and Frank W Nijhoff ${ }^{2, *}$ \\ ${ }^{1}$ School of Mathematics and Statistics, Zhengzhou University, Zhengzhou, 450001, \\ People's Republic of China \\ 2 School of Mathematics, University of Leeds, Leeds LS2 9JT, United Kingdom \\ E-mail: F.W.Nijhoff@leeds.ac.uk
}

Received 26 May 2020, revised 15 January 2021

Accepted for publication 20 January 2021

Published 7 May 2021

\begin{abstract}
The Q1 lattice equation, a member in the Adler-Bobenko-Suris list of 3D consistent lattices, is investigated. By using the multidimensional consistency, a novel Lax pair for Q1 equation is given, which can be nonlinearized to produce integrable symplectic maps. Consequently, a Riemann theta function expression for the discrete potential is derived with the help of the Baker-Akhiezer functions. This expression leads to the algebro-geometric integration of the Q1 lattice equation, based on the commutativity of discrete phase flows generated from the iteration of integrable symplectic maps.
\end{abstract}

Keywords: Baker-Akhiezer functions, algebro-geometric solutions, integrable lattice equations, integrable symplectic maps

Mathematics Subject Classification numbers: 37J10, 37K10, 39A14.

\section{Introduction}

Discrete integrable systems form an increasingly important part of the theory of soliton equations, and it has become a main subject of study within the wider area of integrable systems in recent decades. In particular the study of partial difference equations on a space-time lattice has undergone a dramatic development in parallel to the more familiar theory of integrable partial differential equations, (cf e.g. [1] for an elementary introduction). What has emerged from

*Author to whom any correspondence should be addressed.

Recommended by Dr Irina Nenciu.

(c) (i) Original content from this work may be used under the terms of the Creative Commons Attribution 3.0 licence. Any further distribution of this work must maintain attribution to the author(s) and the title of the work, journal citation and DOI. 
these developments, is on the one hand a remarkable level of analogy between the theory of discrete systems and that of continuous integrable systems (cf e.g. [2]), but at the same time a deepening of understanding of the phenomenon of integrability by exploiting the richness of the discrete world and how it has spawned new mathematical concepts and ideas, cf also [3]. One remarkable breakthrough was the classification of integrable quadrilateral lattice equations, i.e. partial difference equations on a lattice of elementary quadrilaterals on each face of which the equation is defined, and that exhibit as their key integrability feature the aspect of multidimensional consistency (the latter forming a discrete analogue of the existence of infinite hierarchies of partial differential equations of soliton type), cf [1] for details. The result in [4] demonstrated that under certain assumptions, and up to Möbius equivalence, the scalar quadrilateral lattice equations can be classified as comprising only nine equations, denoted as $\mathrm{H} 1, \mathrm{H} 2, \mathrm{H} 3, \mathrm{~A} 1$, A2, and Q1, Q2, Q3, Q4. These are nowadays referred to as the ABS (Adler-Bobenko-Suris) list of quadrilateral lattice equations, and they have been widely studied ever since. Some of these equations, such as the lattice Korteweg-de Vries (KdV) family of equations [5], were already known in the older literature since the early 1980s. They appear as the special value $\delta=0$ case of a parameter that appears in several members of the ABS list, but the list also contains some novel equations, as well as the $\delta \neq 0$ variants, which were new. It is especially for those latter cases that the question of finding explicit solutions has become quite prominent, as they exhibit new complicating features in comparison to the $\delta=0$ cases for which solutions, including rational and soliton solutions as well as algebro-geometric solutions, were already known. While soliton and elliptic type solutions were constructed for all ABS equations in a series of studies [6-8], in this paper we address for the first time the issue of constructing (higher genus) algebro-geometric solutions of one of these novel equations in the ABS list, namely the $(\mathrm{Q} 1)_{\delta}$ for $\delta \neq 0$.

Thus, in this paper we construct algebro-geometric solutions of the Q1 quadrilateral lattice equation,

$$
\Xi^{(0,2)} \equiv \beta_{1}^{2}(\tilde{\bar{u}}-\tilde{u})(\bar{u}-u)-\beta_{2}^{2}(\overline{\tilde{u}}-\bar{u})(\tilde{u}-u)+\delta^{2} \beta_{1}^{2} \beta_{2}^{2}\left(\beta_{1}^{2}-\beta_{2}^{2}\right)=0,
$$

denoted by the symbol $\Xi^{(j, k)}$ where $j, k$ corresponds to the number of continuous and discrete variables respectively. In (1.1) we have adopted our preferred short-hand notation for lattice systems, i.e., partial difference equations for functions $u=u_{m, n}$ depending on two discrete independent variables $m, n \in \mathbb{Z}$, forming a regular lattice with coordinates $(m, n) \in \mathbb{Z}^{2}$, and where elementary lattice shifts are denoted by $\tilde{u}=u_{m+1, n}, \bar{u}=u_{m, n+1}$. Equation (1.1) is a member in the well-known ABS list of 3D consistent lattices [4], where $\beta_{1}, \beta_{2}$ are (lattice) parameters associated with the two lattice directions respectively, while $\delta$ is a fixed parameter. For $\delta \neq 0$ this equation first appeared in the classification of [4], but the case $\delta=0$ (which we will denote by $(\mathrm{Q} 1)_{0}$ ) first appeared in [5] where, due to the appearance of the canonical cross ratio of four variables, namely written in the form

$$
\frac{(\overline{\tilde{u}}-\bar{u})(\tilde{u}-u)}{(\tilde{\bar{u}}-\tilde{u})(\bar{u}-u)}=\frac{\beta_{1}^{2}}{\beta_{2}^{2}}
$$

it was identified as a lattice version of the Schwarzian KdV equation [9, 10],

$$
\frac{u_{y}}{u_{x}}+\frac{1}{4}\left[\frac{u_{x x x}}{u_{x}}-\frac{3}{2}\left(\frac{u_{x x}}{u_{x}}\right)^{2}\right]=0 .
$$

Equation (1.3) is a relative of the famous $\mathrm{KdV}$ equation which is invariant under Möbius transformation. Its lattice version, i.e., the $(\mathrm{Q} 1)_{0}$ lattice, which first appeared in [5], can be used to 
define a discrete conformal map, by which the Riemann theta function solutions to (Q1) $)_{0}$ are calculated $[11,12]$. In the previous papers $[13,14]$, we constructed algebro-geometric solutions of the $(\mathrm{Q} 1)_{0}$ equation, using the method of symplectic maps arising from a nonlinearisation approach $[15,16]$.

The present paper considers the $\delta$-parameter extension of $(\mathrm{Q} 1)_{0}$, which amounts to a significant departure from the $\delta=0$ case, since in a sense it 'lifts' the equation away from the KdV related lattice equations, cf e.g. [1], and towards what one could call the Krichever-Novikov (KN) class, on which we say a bit more below (see section 2). Furthermore, in the soliton solutions, cf [6], the presence of the parameter $\delta$ effectively induces a deformation of the curve on which the lattice parameters (in (1.1) the parameters $\beta_{1}, \beta_{2}$ ) take their values, and hence in that sense the switching on of the $\delta$ represents an unfolding of the corresponding 'parameter curve'. As a consequence of the presence of this parameter also the corresponding Lax pair is significantly more complicated than the KdV type lattice equations [13, $14,17,18]$, and various other simplifying features disappear. Hence, also the nonlinearization approach to the algebro-geometric solutions is significantly different from the one for the $\mathrm{KdV}$ class systems. With regard to explicit solutions of (1.1) relatively little is known so far: soliton solutions were constructed in [6], while elliptic type solutions were presented in [7], along with those of all members of the ABS list apart from the top equation Q4. Elliptic $N$-soliton type solutions of the latter equation (where the equation itself is defined over an elliptic curve) were constructed in [8]. Moreover, in [19], it indicates that the Q1 lattice admits rational solutions. So far, to our knowledge, no explicit results exist about the algebro-geometric solutions expressed in terms of $\theta$-functions corresponding to a hyperelliptic Riemann surface of any of the $\delta$-parameter equations in the ABS list for higher genus $g>1$. The present paper forms the first step towards filling this lacuna in the theory.

The algebro-geometric approach to soliton equations was initiated by early work on the periodic initial value problem for the $\mathrm{KdV}$ and similar equations [20], (and references therein). It basically reduces the problem to that of mapping the initial data to points on the Riemann surface of a higher genus algebraic curve in the space of spectral variables, and using the properties of Abelian functions on that Riemann surface to formulate a (Jacobi type) inverse problem on that Riemann surface and the associated Jacobian of the curve. The results of the inverse problem can usually be expressed in terms of Riemann theta functions associated with the curve. The method we employ in the present paper to solve the Q1 lattice equation (1.1) uses a variant of the standard theory of finite-gap integration based on the construction of integrable symplectic maps in combination with the discrete version of the Liouville-Arnold theory [21-25]. In contrast to the approach presented in [17, 18, 26], which employs associated completely integrable Hamiltonian systems (in terms of an auxiliary continuous time-variable) for the integration of the lattice systems, in the present approach we circumvent the need for an associated continuous variable and use the discrete equation itself as the starting point. This, through the property of multidimensional consistency, cf [1], provides the Lax pair, the commuting matrix operators, as well as the spectral curve associated with a hyperelliptic Riemann surface, in the details of the construction of solutions.

This paper is organised as follows. In section 2, we present the relevant Lax pair as well as the underlying continuous systems associated with the quadrilateral lattice through continuum limits. In section 3, based on two commuting operators, we obtain the relevant symplectic map, whose integrability is proved by using the $r$-matrix and (quasi-) Abel-Jacobi variables. As a consequence, the discrete flows are constructed via the iteration of integrable symplectic maps in section 4. Moreover, the evolution of the potential along the discrete 
flows is inverted with the help of the Baker-Akhiezer functions. As a result, the algebrogeometric integration of the Q1 lattice equation is deduced by the commutativity of two discrete phase flows sharing the same invariants. We finish the paper with some conclusive remarks in section 5 .

\section{Lax pairs and associated continuous equations}

By the method of multidimensional consistency, cf [1], a basic discrete spectral problem is derived directly from equation (1.1)

$$
\tilde{\chi}=\mathcal{D}^{(\beta)}(\lambda ; b) \chi, \quad \mathcal{D}^{(\beta)}(\lambda ; b)=\frac{1}{B}\left(\begin{array}{cc}
\lambda b & \lambda^{2} \delta^{2} \beta+\beta B^{2} \\
\beta & \lambda b
\end{array}\right),
$$

where $B=\left(b^{2}-\delta^{2} \beta^{2}\right)^{1 / 2}, \beta b=\tilde{u}-u$. The factor $B$ is there to avoid that non-trivial relations arise from the determinantal condition of the zero-curvature relation, and one way of guaranteeing that happens is by requiring that $\operatorname{det} \mathcal{D}^{(\beta)}(\lambda ; b)=\lambda^{2}-\beta^{2}$ is a constant. The general form (2.1) of the Lax pair for quadrilateral lattice equations was given in [27], in the context of the one for the generic case of the lattice $\mathrm{KN}$ system, i.e., Q4 lattice equation. The latter contains as a special case the Q1 model, cf also [28]. In the special case of the Q1 lattice equation (1.1) the zero-curvature representation adopts the form

$$
\begin{aligned}
& \tilde{\chi}=\mathcal{D}^{\left(\beta_{1}\right)} \chi \equiv \mathcal{D}^{\left(\beta_{1}\right)}\left(\lambda ; b_{1}\right) \chi, \quad \text { with } \quad b_{1}=(\tilde{u}-u) / \beta_{1}, \\
& \bar{\chi}=\mathcal{D}^{\left(\beta_{2}\right)} \chi \equiv \mathcal{D}^{\left(\beta_{2}\right)}\left(\lambda ; b_{2}\right) \chi, \quad \text { with } \quad b_{2}=(\bar{u}-u) / \beta_{2} \text {. }
\end{aligned}
$$

In fact, computing the difference $\overline{\tilde{\chi}}-\tilde{\bar{\chi}}$ yields the following identity:

$$
\overline{\mathcal{D}}^{\left(\beta_{1}\right)} \mathcal{D}^{\left(\beta_{2}\right)}-\tilde{\mathcal{D}}^{\left(\beta_{2}\right)} \mathcal{D}^{\left(\beta_{1}\right)}=\frac{\Xi^{(0,2)}}{\bar{B}_{1} \tilde{B}_{2} B_{1} B_{2}}\left(\begin{array}{cc}
\lambda^{2} S_{1}+\beta_{1} \beta_{2} \bar{B}_{1} \tilde{B}_{2} S_{0} & \lambda^{2} \delta^{2} S_{2}+\lambda S_{3} \\
\lambda S_{2} & \lambda^{2} S_{1}-\beta_{1} \beta_{2} B_{1} B_{2} S_{0}
\end{array}\right),
$$

in which $B_{k}=\left(b_{k}^{2}-\delta^{2} \beta_{k}^{2}\right)^{1 / 2}$, and where the quantities $S_{j}$ are given by

$$
\begin{aligned}
& S_{0}=-\frac{\tilde{b}_{2} b_{2}+\bar{b}_{1} b_{1}-\delta^{2}\left(\beta_{1}^{2}+\beta_{2}^{2}\right)}{\left(\tilde{B}_{2} B_{2}+\bar{B}_{1} B_{1}\right) \beta_{1}^{2} \beta_{2}^{2}}, \\
& S_{1}=-\frac{\delta^{2}(\overline{\tilde{u}}-\tilde{u}-\bar{u}+u)\left(I_{1} J_{2}+I_{2} J_{1}\right)}{\left(\tilde{B}_{2} B_{1} I_{2}+\bar{B}_{1} B_{2} I_{1}\right) \beta_{1}^{2} \beta_{2}^{2}}, \\
& S_{2}=-\frac{(\overline{\tilde{u}}-\tilde{u}-\bar{u}+u)\left(I_{1} J_{2}+I_{2} J_{1}\right)}{\left(\tilde{B}_{2} B_{1} J_{2}+\bar{B}_{1} B_{2} J_{1}\right) \beta_{1}^{2} \beta_{2}^{2}}, \\
& S_{3}=\frac{\delta^{2}(\overline{\tilde{u}}-\tilde{u}-\bar{u}+u)}{\left(\tilde{B}_{2} B_{1} K_{2}+\bar{B}_{1} B_{2} K_{1}\right) \beta_{1}^{2} \beta_{2}^{2}}\left[\left(I_{1} K_{2}+I_{2} K_{1}\right)\left(\beta_{1}^{2}+\beta_{2}^{2}\right)-\left(J_{1} K_{2}+J_{2} K_{1}\right)(\overline{\tilde{u}}-u)\right],
\end{aligned}
$$

with

$$
\begin{array}{lll}
I_{1}=\tilde{b}_{2} b_{1}+\delta^{2} \beta_{1} \beta_{2}, & J_{1}=\beta_{1} \tilde{b}_{2}+\beta_{2} b_{1}, & K_{1}=\beta_{1} \tilde{b}_{2} B_{1}^{2}+\beta_{2} b_{1} \tilde{B}_{2}^{2}, \\
I_{2}=\bar{b}_{1} b_{2}+\delta^{2} \beta_{1} \beta_{2}, & J_{2}=\beta_{2} \bar{b}_{1}+\beta_{1} b_{2}, & K_{2}=\beta_{2} \bar{b}_{1} B_{2}^{2}+\beta_{1} b_{2} \bar{B}_{1}^{2} .
\end{array}
$$

From equation (2.3) we conclude that the zero-curvature condition (implying $\overline{\tilde{\chi}}=\tilde{\chi}$ ) is satisfied iff $\Xi^{(0,2)}=0$, i.e. if the Q1 lattice equation (1.1) holds for the function $u$, since generically 
at least one of the entries in the matrix on the right-hand side of (2.3) is nonzero. Thus, the relation (2.3) forms a bridge between the discrete zero-curvature equation and the Q1 model.

Let us now consider continuum limits of the Q1 equation. A special sequel of continuum limits yield the following form for a semi-continuum and full continuum limit respectively,

$$
\begin{aligned}
& \Xi^{(1,1)} \equiv \beta_{1}^{2} \tilde{u}_{x} u_{x}-(\tilde{u}-u)^{2}+\delta^{2} \beta_{1}^{4}=0, \\
& \Xi^{(2,0)} \equiv u_{y}+\frac{1}{4}\left(u_{x x x}-\frac{3}{2} \frac{u_{x x}^{2}-4 \delta^{2}}{u_{x}}\right)=0 .
\end{aligned}
$$

In fact, setting $\beta_{2}=c_{2} \varepsilon$, and $\bar{u}(x, y)=u\left(x+\beta_{2}, y-\beta_{2}^{3} / 3\right)$, then as remains $\beta_{1}$ constant while $\beta_{2} \rightarrow 0$, i.e. $\varepsilon \rightarrow 0$, we have the Taylor expansion

$$
\Xi^{(0,2)} \equiv c_{2}^{2} \varepsilon^{2} \Xi^{(1,1)}+O\left(\varepsilon^{3}\right) .
$$

Furthermore, setting $\beta_{k}=c_{k} \varepsilon$, and $\tilde{u}(x, y)=u\left(x+\beta_{1}, y-\beta_{1}^{3} / 3\right)$, and $\bar{u}(x, y)=u\left(x+\beta_{2}, y-\right.$ $\left.\beta_{2}^{3} / 3\right)$. As $\varepsilon \rightarrow 0$, we obtain

$$
\Xi^{(0,2)} \equiv \frac{2}{3} u_{x}\left(c_{1}^{2}-c_{2}^{2}\right) \varepsilon^{2} \Xi^{(2,0)}+O\left(\varepsilon^{3}\right)
$$

We note that equation (2.5) with $\delta=0$ is exactly the Schwarzian KdV equation (1.3) which is the continuum limit of $(\mathrm{Q} 1)_{0}$. Moreover, equation (2.5) is a special case of the KN equation [9], which in its most general form is given by

$$
u_{y}=u_{x x x}-\frac{3}{2 u_{x}}\left(u_{x x}^{2}-r(u)\right)+c u_{x}, \quad r^{(5)}=0
$$

with $r(u)$ an arbitrary quadratic polynomial with constant coefficients. Adler [29], discovered the following Bäcklund transformation for (2.6):

$$
u_{x} v_{x}=h(u, v)
$$

where $h(u, v)$ is an arbitrary symmetric biquadratic polynomial, i.e., a polynomial of degree less than 3 in each variable. Furthermore, the nonlinear superposition principle for (2.7), i.e., the permutability condition of the Bäcklund transform, in the most general, elliptic, form gives rise to the Q4 lattice equation [28, 29]. In the more special case of the Q1 equation (2.7) coincides with the semicontinuous equation (2.4) written as

$$
u_{x} \tilde{u}_{x}=\beta_{1}^{-2}(\tilde{u}-u)^{2}-\delta^{2} \beta_{1}^{2},
$$

by identifying $v=\tilde{u}$. The observation that the Bäcklund transformed quantity $\tilde{u}$ can be identified with a lattice shift goes back to $[30,31]$. On the level of the Lax pair, the semi-discrete zero curvature equation $\Lambda=\mathcal{D}_{x}^{(\beta)}-\tilde{\mathcal{U}} \mathcal{D}^{(\beta)}+\mathcal{D}^{(\beta)} \mathcal{U}=0$, with $\mathcal{U}$ given by

$$
\partial_{x} \chi=\mathcal{U}(\lambda ; u) \chi=\frac{1}{\lambda u_{x}}\left(\begin{array}{cc}
0 & \lambda^{2} \delta^{2}+u_{x}^{2} \\
1 & 0
\end{array}\right) \chi
$$

gives rise to the semi-discrete equation (2.4) as commutability condition. Indeed, by direct calculations we have

$$
\Lambda=\frac{-\Xi^{(1,1)}}{\beta_{1} B_{1} \tilde{u}_{x} u_{x}}\left[\frac{b_{1, x}}{B_{1}^{2}}\left(\begin{array}{cc}
\lambda \delta^{2} \beta_{1} & \lambda^{2} \delta^{2} b_{1} \\
b_{1} & \lambda \delta^{2} \beta_{1}
\end{array}\right)+\frac{1}{\lambda}\left(\begin{array}{cc}
\tilde{u}_{x} & 0 \\
0 & -u_{x}
\end{array}\right)\right] .
$$


We mention in passing that the KN equation (2.6) arose in [9] from the problem of finding special finite-gap solutions of the Kadomtsev-Petviashvili equation associated with rank 2 holomorphic vector bundles. We mention also that already early on it was observed that the semi-discrete system is perfectly adapted for performing numerical calculations, and for producing the plots of solutions, which reveals that the algebro-geometric solutions have a practical value as standard special functions as well [32-34].

Motivated by these known facts, this paper is dedicated to the problem of constructing the algebro-geometric integration for the Q1 equation (1.1). To set up the finite-gap scheme for Q1 it is most convenient to perform the calculations from a Lax representation perspective. In the development of this method, a fundamental role is played by the Burchnall-Chaundy theory of commuting differential operators $[35,36]$, whose discrete analogue concerning commutative rings of periodic difference operators was developed [37, 38]. Their common eigenfunctions are vector bundles over a Riemann surface defined by the corresponding eigenvalues, and this forms the underlying geometry for the reconstruction of the potentials from a special class of spectral functions which themselves can be obtained from solving a classical Jacobi inversion problem for Abelian integrals on hyperelliptic Riemann surfaces [39-41]. Furthermore, the algebro-geometric solutions of the associated discrete systems can be expressed in terms of the Riemann theta functions associated with the Riemann surface, and a suitable choice of a homology basis of curves (see [17, 42-50] and the reference therein). The approach in this paper for the solution of the discrete equation is based on the construction of commuting integrable symplectic (dynamical) maps, which in turn can be resolved in terms of the algebro-geometric data.

\section{Construction of the nonlinear integrable symplectic map}

In this section we discuss how a linear map can be nonlinearized to produce a nonlinear integrable symplectic map on the symplectic manifold $\mathcal{N}=\left(\mathbb{R}^{2 N}, \mathrm{~d} p \wedge \mathrm{d} q\right)$ with associated coordinates $(p, q)=\left(p_{1}, \ldots, p_{N}, q_{1}, \ldots, q_{N}\right)^{T}$, where $N$ is a positive integer. A map $\mathcal{S}:(p, q) \mapsto$ $(\tilde{p}, \tilde{q})$ is a symplectic transformation [51-53], if $\mathcal{S}^{*}(\mathrm{~d} p \wedge \mathrm{d} q)=\mathrm{d} p \wedge \mathrm{d} q$. A well-defined notion of integrability for symplectic maps was first given by Veselov [22, 23]. It is entirely analogous to that of the Liouville-Arnold in the continuous-time case, i.e., there exists $N$ smooth functions $I_{1}, \ldots, I_{N}: \mathbb{R}^{2 N} \rightarrow \mathbb{R}$ with the following properties:

(a) The functions are invariants of the map, i.e., $\mathcal{S}^{*} I_{j}=I_{j}$.

(b) The invariant functions are in involution with respect to the Poisson bracket, i.e., $\left\{I_{i}, I_{j}\right\}=0$.

(c) The invariant functions are functionally independent throughout the phase space.

To construct an integrable nonlinear symplectic map suitable for the integration of the Q1 equation, we use the nonlinearisation method of $[17,18,26]$ and apply it to the linear map associated with the spectral problem (2.1) of Q1, which is given by:

$$
\left(\begin{array}{c}
\tilde{p}_{j} \\
\tilde{q}_{j}
\end{array}\right)=\left(\alpha_{j}^{2}-\beta^{2}\right)^{-1 / 2} \mathcal{D}^{(\beta)}\left(\alpha_{j} ; b\right)\left(\begin{array}{c}
p_{j} \\
q_{j}
\end{array}\right), \quad 1 \leqslant j \leqslant N
$$

where $\left\{\alpha_{j}\right\}_{j=1}^{N}$ are the corresponding eigenvalues. We assume, moreover, that these eigenvalues $\alpha_{1}^{2}, \ldots, \alpha_{N}^{2}$ are mutually distinct and non-zero. It turns out that the linear map (3.1) can be extended to a nonlinear integrable symplectic map by imposing a restriction on the discrete 
potential $^{3}$ which is the quantity $b$. This fact has led in several cases to the construction of integrable symplectic maps in the process of constructing the finite gap solutions for several discrete soliton equations $[17,18,26]$. In the present case, the operator associated with the Lax pair (2.2) is the Darboux matrix $\mathcal{D}^{(\beta)}(\lambda ; b)$ given by (2.1). Through direct computation, we find that there is a matrix operator, i.e., the Lax matrix

$$
\mathcal{L}(\lambda ; p, q)=\left(\begin{array}{cc}
0 & \delta^{2}\left(\lambda^{2}-\langle A q, q\rangle\right) \\
1 & 0
\end{array}\right)+\left(\begin{array}{cc}
\lambda Q_{\lambda}(p, q) & -Q_{\lambda}(A p, p) \\
Q_{\lambda}(A q, q) & -\lambda Q_{\lambda}(p, q)
\end{array}\right)
$$

that commutes with $\mathcal{D}^{(\beta)}(\lambda ; b)$ :

$$
\mathcal{L}(\lambda ; \tilde{p}, \tilde{q}) \mathcal{D}^{(\beta)}(\lambda ; b)-\mathcal{D}^{(\beta)}(\lambda ; b) \mathcal{L}(\lambda ; p, q)=0,
$$

where $A=\operatorname{diag}\left(\alpha_{1}, \ldots, \alpha_{N}\right),\langle\xi, \eta\rangle=\Sigma_{j=1}^{N} \xi_{j} \eta_{j}$ and $Q_{\lambda}(\xi, \eta)=\left\langle\left(\lambda^{2}-A^{2}\right)^{-1} \xi, \eta\right\rangle$. This commutative relation (3.3) in cases considered implies a quadratic equation providing the constraints on discrete potentials $[17,18,26]$.

Proposition 3.1. The constraint for the discrete potential $b$ in the spectral problem (2.1) satisfies

$$
P^{(\beta)}(b ; p, q) \equiv b^{2} \mathcal{L}^{21}(\beta ; p, q)+2 b \mathcal{L}^{11}(\beta ; p, q)-\mathcal{L}^{12}(\beta ; p, q)=0 .
$$

Proof. First, note that the Lax matrix (3.2) can be rewritten as

$$
\begin{aligned}
& \mathcal{L}(\lambda ; p, q)=\left(\begin{array}{cc}
0 & \delta^{2}\left(\lambda^{2}-\langle A q, q\rangle\right) \\
1 & 0
\end{array}\right)+\frac{1}{2} \sum_{j=1}^{N}\left(\frac{\varepsilon_{j}}{\lambda-\alpha_{j}}+\frac{\sigma_{3} \varepsilon_{j} \sigma_{3}}{\lambda+\alpha_{j}}\right), \\
& \varepsilon_{j}=\left(\begin{array}{cc}
p_{j} q_{j} & -p_{j}^{2} \\
q_{j}^{2} & -p_{j} q_{j}
\end{array}\right) .
\end{aligned}
$$

Then we calculate $\mathcal{L}(\lambda ; \tilde{p}, \tilde{q}) \mathcal{D}^{(\beta)}(\lambda ; b)-\mathcal{D}^{(\beta)}(\lambda ; b) \mathcal{L}(\lambda ; p, q)=I+I I$,

$$
\begin{aligned}
I & =\left(\begin{array}{cc}
\frac{-\beta}{B}\left(\delta^{2}\langle A \tilde{q}, \tilde{q}\rangle+B^{2}\right) & -\lambda \delta^{2}(\langle A \tilde{q}, \tilde{q}\rangle-\langle A q, q\rangle) \\
0 & \frac{\beta}{B}\left(\delta^{2}\langle A q, q\rangle+B^{2}\right)
\end{array}\right), \\
I I & =\frac{1}{2} \sum_{j=1}^{N} \frac{\tilde{\varepsilon}_{j} \mathcal{D}^{(\beta)}(\lambda ; b)-\mathcal{D}^{(\beta)}(\lambda ; b) \varepsilon_{j}}{\lambda-\alpha_{j}}+\frac{\sigma_{3} \tilde{\varepsilon}_{j} \sigma_{3} \mathcal{D}^{(\beta)}(\lambda ; b)-\mathcal{D}^{(\beta)}(\lambda ; b) \sigma_{3} \varepsilon_{j} \sigma_{3}}{\lambda+\alpha_{j}} \\
& =\frac{b}{B}(\langle\tilde{p}, \tilde{q}\rangle-\langle p, q\rangle) \sigma_{3}+\frac{\beta \delta^{2}}{B}\left(\begin{array}{cc}
-\langle A q, q\rangle & \lambda(\langle\tilde{p}, \tilde{q}\rangle+\langle p, q\rangle)), \\
0 & \langle A \tilde{q}, \tilde{q}\rangle
\end{array}\right),
\end{aligned}
$$

where we use $\tilde{\varepsilon}_{j} \mathcal{D}^{(\beta)}\left(\alpha_{j} ; b\right)=\mathcal{D}^{(\beta)}\left(\alpha_{j} ; b\right) \varepsilon_{j}, \sigma_{3} \mathcal{D}^{(\beta)}\left(\alpha_{j} ; b\right) \sigma_{3}=-\mathcal{D}^{(\beta)}\left(-\alpha_{j} ; b\right)$. From equations (2.1) and (3.1), we have

$$
\tilde{p}=\left(A^{2}-\beta^{2}\right)^{-1 / 2} \frac{1}{B}\left(b A p+\beta \delta^{2} A^{2} q+\beta B^{2} q\right),
$$

\footnotetext{
${ }^{3}$ This assertion is based on the observation, going back to [9], that there exists an operator commuting with a member in the Lax representation for nonlinear equations of $\mathrm{KdV}$ type, when discussing the associated finite gap classes of exact solutions.
} 


$$
\tilde{q}=\left(A^{2}-\beta^{2}\right)^{-1 / 2} \frac{1}{B}(\beta p+b A q)
$$

Hence

$$
\begin{aligned}
\mathcal{L}(\lambda ; \tilde{p}, \tilde{q}) \mathcal{D}^{(\beta)}(\lambda ; b)-\mathcal{D}^{(\beta)}(\lambda ; b) \mathcal{L}(\lambda ; p, q) \\
\quad=\frac{1}{B}\left[b(\langle\tilde{p}, \tilde{q}\rangle-\langle p, q\rangle)-\beta \delta^{2}(\langle A \tilde{q}, \tilde{q}\rangle+\langle A q, q\rangle)-\beta B^{2}\right] \sigma_{3} \\
\quad=-\frac{\beta}{B} P^{(\beta)}(b ; p, q) \sigma_{3},
\end{aligned}
$$

where $\sigma_{3}$ is the usual Pauli matrix. This concludes the proof by using equation (3.3).

Following the spirit of [9], we use the Burchnall-Chaundy theory [35-38] to study the commuting operators $\mathcal{L}(\lambda ; p, q)$ and $\mathcal{D}^{(\beta)}(\lambda ; b)$. We shall investigate their common eigenfunctions and the corresponding eigenvalues which lie on a Riemann surface. In our case, the operator $\mathcal{L}(\lambda ; p, q)$ has two eigenvalues,

$$
\pm \mathcal{H}_{\lambda}= \pm \sqrt{-\mathcal{F}_{\lambda}}
$$

where

$$
\begin{aligned}
\mathcal{F}_{\lambda}= & \mathcal{F}_{\lambda}(p, q) \triangleq \operatorname{det} \mathcal{L}(\lambda ; p, q) \\
= & \left(-\delta^{2} \lambda^{2}+\delta^{2}\langle A q, q\rangle+Q_{\lambda}(A p, p)\right)\left(1+Q_{\lambda}(A q, q)\right)-\lambda^{2} Q_{\lambda}^{2}(p, q) \\
= & -\delta^{2} \lambda^{2}+Q_{\lambda}(A p, p)-\delta^{2} Q_{\lambda}\left(A^{3} q, q\right)+\delta^{2}\langle A q, q\rangle Q_{\lambda}(A q, q) \\
& +Q_{\lambda}(A p, p) Q_{\lambda}(A q, q)-\lambda^{2} Q_{\lambda}^{2}(p, q),
\end{aligned}
$$

with $Q_{\lambda}\left(A^{3} q, q\right)=\lambda^{2} Q_{\lambda}(A q, q)-\langle A q, q\rangle$. Moreover, $\mathcal{F}_{\lambda}$ is a rational function of $\zeta=\lambda^{2}$, and has simple pole at each point $\alpha_{j}^{2}$. Inspired by the relation between the eigenvalues $\mathcal{H}_{\lambda}$ and $\lambda$, given by (3.5), we consider the factorization of $\mathcal{F}_{\lambda}$

$$
\mathcal{F}_{\lambda}=-\delta^{2} \frac{\prod_{j=1}^{N+1}\left(\zeta-\lambda_{j}^{2}\right)}{\alpha(\zeta)}=-\delta^{2} \frac{R(\zeta)}{\zeta \alpha^{2}(\zeta)}, \quad \alpha(\zeta)=\Pi_{k=1}^{N}\left(\zeta-\alpha_{k}^{2}\right)
$$

and then construct a hyperelliptic spectral curve associated with a two-sheeted Riemann surface of genus $g=N$, cf [54-56],

$$
\mathcal{R}: \xi^{2}=R(\zeta)
$$

Since $\operatorname{deg} R=2 N+2$, the above curve (3.8) has two infinities $\infty_{+}, \infty_{-}$. For any $\zeta \in \mathbb{C}$, in the non-branch case (not equal to $\lambda_{j}^{2}, \alpha_{j}^{2}$ or 0 ) we call the collection of points on $\mathcal{R}$ of the form

$$
\mathfrak{p}(\zeta)=(\zeta, \xi=\sqrt{R(\zeta)}), \quad(\tau \mathfrak{p})(\zeta)=(\zeta, \xi=-\sqrt{R(\zeta)})
$$

the upper and lower sheets, respectively, where $\tau: \mathcal{R} \rightarrow \mathcal{R}$ is the map of changing sheets. The branch point, given by $\zeta=0$ and $\xi=0$, is denoted by $\mathfrak{o}$.

To proceed with the actual integration of the map, we first list some basic objects on $\mathcal{R}$ [54-56]. Let $a_{1}, \ldots, a_{g}, b_{1}, \ldots, b_{g}$ be the canonical basis of the homology group $H_{1}(\mathcal{R})$ and 
$\omega_{1}^{\prime}, \ldots, \omega_{g}^{\prime}$ be the basis of holomorphic differentials:

$$
\omega_{l}^{\prime}=\sum_{s=1}^{g} \frac{\zeta^{g-s} \mathrm{~d} \zeta}{2 \sqrt{R(\zeta)}}, \quad 1 \leqslant l \leqslant g
$$

whose integral along $a_{k}$ is denoted by $a_{l k}$. Then (3.9) in the vector form $\vec{\omega}^{\prime}=\left(\omega_{1}^{\prime}, \ldots, \omega_{g}^{\prime}\right)^{T}$ can be normalized into $\vec{\omega}=\left(\omega_{1}, \ldots, \omega_{g}\right)^{T}=C \vec{\omega}^{\prime}$ with $C=\left(a_{l k}\right)_{g \times g}^{-1}$.

Periodic vectors $\vec{\delta}_{k}, \vec{B}_{k}$ are defined as integrals of $\vec{\omega}$ along $a_{k}, b_{k}$, respectively. They span a lattice $\mathscr{T}$, which defines the Jacobian variety $J(\mathcal{R})=\mathbb{C}^{g} / \mathscr{T}$. The matrix $B=\left(\vec{B}_{1}, \ldots, \vec{B}_{g}\right)$ is used to construct the Riemann theta functions which will be used in section 4 ,

$$
\theta(z, B)=\sum_{z^{\prime} \in \mathbb{Z}^{g}} \exp \pi \sqrt{-1}\left(\left\langle B z^{\prime}, z^{\prime}\right\rangle+2\left\langle z, z^{\prime}\right\rangle\right), \quad z \in \mathbb{C}^{g} .
$$

The Abel map $\mathcal{A}: \operatorname{Div}(\mathcal{R}) \rightarrow J(\mathcal{R})$ defined as

$$
\mathscr{A}(\mathfrak{p})=\int_{\mathfrak{p}_{0}}^{\mathfrak{p}} \vec{\omega}, \quad \mathscr{A}\left(\Sigma n_{k} \mathfrak{p}_{k}\right)=\Sigma n_{k} \mathscr{A}\left(\mathfrak{p}_{k}\right),
$$

is the key ingredient in the Jacobi inversion problem.

As a consequence we obtain a nonlinear map arising from the linear map (3.1),

$$
\mathcal{S}_{\beta}:\left(\begin{array}{c}
\tilde{p} \\
\tilde{q}
\end{array}\right)=\left.\left(A^{2}-\beta^{2}\right)^{-1 / 2} \frac{1}{B}\left(\begin{array}{c}
b A p+\beta \delta^{2} A^{2} q+\beta B^{2} q \\
\beta p+b A q
\end{array}\right)\right|_{b=f_{\beta}(p, q)},
$$

where $b=f_{\beta}(p, q)$ is given by the roots of quadratic equation (3.4),

$$
b=f_{\beta}(p, q)=\frac{1}{1+Q_{\beta}(A q, q)}\left(-\beta Q_{\beta}(p, q) \pm \mathcal{H}_{\beta}\right),
$$

which is single-valued as a function of $\mathfrak{p}\left(\beta^{2}\right) \in \mathcal{R}$. Actually, $\beta b$ are the values of the following meromorphic function on the curve $\mathcal{R}$ :

$$
\mathfrak{b}(\mathfrak{p})=\frac{1}{1+Q_{\beta}(A q, q)}\left(-\beta^{2} Q_{\beta}(p, q)+\frac{\delta \xi}{\alpha\left(\beta^{2}\right)}\right),
$$

at the points $\mathfrak{p}\left(\beta^{2}\right)$ and $(\tau \mathfrak{p})\left(\beta^{2}\right)$, respectively. Hence, the nonlinear map $\mathcal{S}_{\beta}$ is well-defined.

The next step is to show that $\mathcal{S}_{\beta}$ given by (3.12) is an integrable symplectic map on $\mathcal{N}=\left(\mathbb{R}^{2 N}, \mathrm{~d} p \wedge \mathrm{d} q\right)$. In order to get the symplecticity of $\mathcal{S}_{\beta}$, we calculate

$$
\begin{aligned}
\left(\begin{array}{l}
\mathrm{d} \tilde{p}_{j} \\
\mathrm{~d} \tilde{q}_{j}
\end{array}\right)= & \left(\alpha_{j}^{2}-\beta^{2}\right)^{-1 / 2}\left(\mathcal{D}^{(\beta)}\left(\alpha_{j} ; b\right)\left(\begin{array}{l}
\mathrm{d} p_{j} \\
\mathrm{~d} q_{j}
\end{array}\right)+\mathcal{C}^{(\beta)}\left(\alpha_{j} ; b\right)\left(\begin{array}{l}
\mathrm{d} p_{j} \\
\mathrm{~d} q_{j}
\end{array}\right) \mathrm{d} b\right), \\
& 1 \leqslant j \leqslant N,
\end{aligned}
$$

where

$$
\mathcal{C}^{(\beta)}\left(\alpha_{j} ; b\right)=\frac{\mathrm{d}}{\mathrm{d} b} \mathcal{D}^{(\beta)}\left(\alpha_{j} ; b\right)=-\frac{b}{B^{2}} \mathcal{D}^{(\beta)}\left(\alpha_{j} ; b\right)+\frac{1}{B}\left(\begin{array}{cc}
\alpha_{j} & 2 \beta b \\
0 & \alpha_{j}
\end{array}\right)
$$


by using equation (3.12). Then,

$$
\sum_{j=1}^{N}\left(\mathrm{~d} \tilde{p}_{j} \wedge \mathrm{d} \tilde{q}_{j}-\mathrm{d} p_{j} \wedge \mathrm{d} q_{j}\right)=\frac{\beta}{2 B^{2}} \mathrm{~d} P^{(\beta)}(b ; p, q) \wedge \mathrm{d} b,
$$

which implies $\mathcal{S}_{\beta}^{*}(\mathrm{~d} p \wedge \mathrm{d} q)=\mathrm{d} \tilde{p} \wedge \mathrm{d} \tilde{q}=\mathrm{d} p \wedge \mathrm{d} q$, since $b=f_{\beta}(p, q)$ given by equation (3.13) satisfies the quadratic equation (3.4). Hence, $\mathcal{S}_{\beta}$ is a symplectic map on $\mathcal{N}=\left(\mathbb{R}^{2 N}, \mathrm{~d} p \wedge \mathrm{d} q\right)$.

Having asserted the symplecticity of the map, we now turn to the construction of its invariant. In fact, by taking the determinant on equation (3.3), we have $\operatorname{det} \mathcal{L}(\lambda ; \tilde{p}, \tilde{q})=$ $\operatorname{det} \mathcal{L}(\lambda ; p, q)$, i.e.,

$$
\tilde{\mathcal{F}}_{\lambda}=\mathcal{F}_{\lambda}
$$

Thus, invariants of the map $\mathcal{S}_{\beta}$ can be generated by the partial fraction expansion,

$$
\mathcal{F}_{\lambda}=-\delta^{2} \lambda^{2}+\sum_{k=1}^{N} \frac{E_{k}}{\lambda^{2}-\alpha_{k}^{2}}
$$

where

$$
\begin{aligned}
E_{k}= & \alpha_{k} p_{k}^{2}-\delta^{2} \alpha_{k}^{3} q_{k}^{2}+\delta^{2}\langle A q, q\rangle \alpha_{k} q_{k}^{2}-p_{k}^{2} q_{k}^{2} \\
& +\frac{\alpha_{k}}{2} \sum_{1 \leqslant j \leqslant N ; j \neq k} \frac{\left(p_{k} q_{j}-p_{j} q_{k}\right)^{2}}{\alpha_{k}^{2}-\alpha_{j}^{2}}-\frac{\left(p_{k} q_{j}+p_{j} q_{k}\right)^{2}}{\alpha_{k}^{2}+\alpha_{j}^{2}} .
\end{aligned}
$$

In fact, substituting (3.17) into the both sides of equation (3.16) and comparing the residues at $\alpha_{k}^{2}$, we obtain

$$
\tilde{E}_{k}=E_{k}, \quad 1 \leqslant k \leqslant N
$$

The next step is to show that $N$ invariant functions $E_{1}, \ldots, E_{N}$ on the phase space $\mathbb{R}^{2 N}$ for the symplectic map $\mathcal{S}_{\beta}$ are in involution with respect to the symplectic structure, and are functionally independent. These conditions are essential conditions to assert the Liouville integrability of symplectic maps. We employ a different approach here from previous papers $[17,18,26]$, by employing an $r$-matrix structure to exhibit the validity of these conditions. The relevant $r$-matrix structure is similar to the well known cases $[2,57,58]$, and through direct calculation we find that the Lax matrix obeys the fundamental Poisson bracket

$$
\{\mathcal{L}(\lambda) \otimes \mathcal{L}(\mu)\}=\left[r(\lambda, \mu), \mathcal{L}_{1}(\lambda)\right]+\left[r^{\prime}(\lambda, \mu), \mathcal{L}_{2}(\mu)\right],
$$

where $\mathcal{L}(\lambda ; p, q)$ is often written as $\mathcal{L}(\lambda)$ for short, $\mathcal{L}_{1}(\lambda)=\mathcal{L}(\lambda) \otimes I, \mathcal{L}_{2}(\mu)=I \otimes \mathcal{L}(\mu)$, and $r^{\prime}(\lambda, \mu)=-r(\mu, \lambda)$ satisfies

$$
\begin{aligned}
r(\lambda, \mu) & =\frac{1}{\lambda^{2}-\mu^{2}}\left(\lambda\left(\sigma_{1} \otimes \sigma_{1}+\sigma_{2} \otimes \sigma_{2}\right)+\mu\left(\sigma_{3} \otimes \sigma_{3}+I\right)\right)+2 \delta^{2} \lambda \sigma_{+} \otimes \sigma_{+} \\
& =\frac{2}{\lambda^{2}-\mu^{2}}\left(\begin{array}{cccc}
\mu & 0 & 0 & 0 \\
0 & 0 & \lambda & 0 \\
0 & \lambda & 0 & 0 \\
0 & 0 & 0 & \mu
\end{array}\right)+2 \delta^{2} \lambda\left(\begin{array}{cccc}
0 & 0 & 0 & 1 \\
0 & 0 & 0 & 0 \\
0 & 0 & 0 & 0 \\
0 & 0 & 0 & 0
\end{array}\right),
\end{aligned}
$$

with $\sigma_{1}, \sigma_{2}, \sigma_{3}, \sigma_{+}$the Pauli matrices and $I$ the usual unit matrix. 
In addition to (3.20), there are two further matrix functions $s, s^{\prime}$ such that

$$
\left\{\mathcal{L}^{2}(\lambda) \otimes \mathcal{L}^{2}(\mu)\right\}=\left[s, \mathcal{L}_{1}(\lambda)\right]+\left[s^{\prime}, \mathcal{L}_{2}(\mu)\right] .
$$

This is derived from

$$
\begin{aligned}
\left\{\mathcal{L}^{2}(\lambda) \otimes \mathcal{L}^{2}(\mu)\right\}= & \mathcal{L}_{1}(\lambda) \mathcal{L}_{2}(\mu)\{\mathcal{L}(\lambda) \otimes \mathcal{L}(\mu)\}+\mathcal{L}_{1}(\lambda)\{\mathcal{L}(\lambda) \otimes \mathcal{L}(\mu)\} \mathcal{L}_{2}(\mu) \\
& +\mathcal{L}_{2}(\mu)\{\mathcal{L}(\lambda) \otimes \mathcal{L}(\mu)\} \mathcal{L}_{1}(\lambda)+\{\mathcal{L}(\lambda) \otimes \mathcal{L}(\mu)\} \mathcal{L}_{2}(\mu) \mathcal{L}_{1}(\lambda)
\end{aligned}
$$

Substituting (3.20) into (3.23) and by using the formula $\mathcal{L}_{1}(\lambda) \mathcal{L}_{2}(\mu)=\mathcal{L}_{2}(\mu) \mathcal{L}_{1}(\lambda)=\mathcal{L}(\lambda) \otimes$ $\mathcal{L}(\mu)$, we get

$$
\begin{aligned}
& s=\mathcal{L}_{1}(\lambda) \mathcal{L}_{2}(\mu) r(\lambda, \mu)+\mathcal{L}_{1}(\lambda) r(\lambda, \mu) \mathcal{L}_{2}(\mu)+\mathcal{L}_{2}(\mu) r(\lambda, \mu) \mathcal{L}_{1}(\lambda)+r(\lambda, \mu) \mathcal{L}_{2}(\mu) \mathcal{L}_{1}(\lambda), \\
& s^{\prime}=\mathcal{L}_{1}(\lambda) \mathcal{L}_{2}(\mu) r^{\prime}(\lambda, \mu)+\mathcal{L}_{1}(\lambda) r^{\prime}(\lambda, \mu) \mathcal{L}_{2}(\mu)+\mathcal{L}_{2}(\mu) r^{\prime}(\lambda, \mu) \mathcal{L}_{1}(\lambda)+r^{\prime}(\lambda, \mu) \mathcal{L}_{2}(\mu) \mathcal{L}_{1}(\lambda) .
\end{aligned}
$$

As a consequence of the $r$-matrix structure we have the following:

Proposition 3.2. The invariants $E_{1}, \ldots, E_{N}$ of the symplectic map $\mathcal{S}_{\beta}$ are in pairwise involution.

Proof. Since $\mathcal{L}^{2}(\lambda)=-\mathcal{F}_{\lambda} I, \mathcal{L}^{2}(\mu)=-\mathcal{F}_{\mu} I$, we calculate

$$
\left\{\mathcal{F}_{\lambda}, \mathcal{F}_{\mu}\right\}=\frac{1}{4} \operatorname{tr}\left\{\mathcal{L}^{2}(\lambda) \otimes \mathcal{L}^{2}(\mu)\right\}
$$

Hence by equation (3.22), we obtain

$$
\left\{\mathcal{F}_{\lambda}, \mathcal{F}_{\mu}\right\}=0, \quad \forall \lambda, \mu \in \mathbb{C} .
$$

Substitute the partial fraction expansion (3.17) into (3.25), then calculate the residues, we have

$$
\left\{E_{k}, E_{j}\right\}=0, \quad 1 \leqslant j, k \leqslant N,
$$

which implies $\left\{E_{k}\right\}$ given by equation (3.18) are in involution.

Interestingly, by the $r$-matrix method, we can get the evolution of the Lax matrix along a phase flow resulting in the independence for invariant functions $[17,59]$. In order to do this we calculate

$$
\frac{\mathrm{d}}{\mathrm{d} t_{\lambda}}\left(\begin{array}{c}
p_{j} \\
q_{j}
\end{array}\right)=\left(\begin{array}{c}
-\partial \mathcal{F}_{\lambda} / \partial q_{j} \\
\partial \mathcal{F}_{\lambda} / \partial p_{j}
\end{array}\right)=\mathcal{W}\left(\lambda, \alpha_{j}\right)\left(\begin{array}{c}
p_{j} \\
q_{j}
\end{array}\right),
$$

where $t_{\lambda}$ is the flow variable corresponding to the Hamiltonian function $\mathcal{F}_{\lambda}$, then we obtain

$$
\mathcal{W}(\lambda, \mu)=\frac{2}{\lambda^{2}-\mu^{2}}\left(\begin{array}{cc}
\lambda \mathcal{L}^{11}(\lambda) & \mu \mathcal{L}^{12}(\lambda) \\
\mu \mathcal{L}^{21}(\lambda) & -\lambda \mathcal{L}^{11}(\lambda)
\end{array}\right)-2 \delta^{2} \mu \mathcal{L}^{21}(\lambda) \sigma_{+} .
$$

This statement can be cast in Lax form as follows:

Lemma 3.1. The Lax matrix $\mathcal{L}(\mu)$ satisfies the evolution equation along the $t_{\lambda}$-flow,

$$
\mathrm{d} \mathcal{L}(\mu) / \mathrm{d} t_{\lambda}=[\mathcal{W}(\lambda, \mu), \mathcal{L}(\mu)] .
$$


Proof. Since $\mathcal{L}^{2}(\lambda)=-\mathcal{F}_{\lambda} I$, we obtain

$$
\begin{aligned}
\left\{\mathcal{L}^{2}(\lambda) \otimes \mathcal{L}(\mu)\right\} & =\left\{-\mathcal{F}_{\lambda} I \otimes \mathcal{L}(\mu)\right\} \\
& =\left(\begin{array}{cc}
-\left\{\mathcal{F}_{\lambda}, \mathcal{L}(\mu)\right\} & 0 \\
0 & -\left\{\mathcal{F}_{\lambda}, \mathcal{L}(\mu)\right\}
\end{array}\right) \\
& =\left(\begin{array}{cc}
\mathrm{d} \mathcal{L}(\mu) / \mathrm{d} t_{\lambda} & 0 \\
0 & \mathrm{~d} \mathcal{L}(\mu) / \mathrm{d} t_{\lambda}
\end{array}\right) .
\end{aligned}
$$

By equation (3.20), we calculate the left-hand side of (3.30) again and get

$$
\begin{aligned}
\left\{\mathcal{L}^{2}(\lambda) \otimes \mathcal{L}(\mu)\right\}= & \mathcal{L}_{1}(\lambda)\{\mathcal{L}(\lambda) \otimes \mathcal{L}(\mu)\}+\{\mathcal{L}(\lambda) \otimes \mathcal{L}(\mu)\} \mathcal{L}_{1}(\lambda) \\
= & \mathcal{L}_{1}(\lambda) r^{\prime}(\lambda, \mu) \mathcal{L}_{2}(\mu)-\mathcal{L}_{1}(\lambda) \mathcal{L}_{2}(\mu) r^{\prime}(\lambda, \mu) \\
& +r^{\prime}(\lambda, \mu) \mathcal{L}_{2}(\mu) \mathcal{L}_{1}(\lambda)-\mathcal{L}_{2}(\mu) r^{\prime}(\lambda, \mu) \mathcal{L}_{1}(\lambda) \\
= & {\left[\mathcal{L}_{1}(\lambda) r^{\prime}(\lambda, \mu)+r^{\prime}(\lambda, \mu) \mathcal{L}_{1}(\lambda), \mathcal{L}_{2}(\mu)\right] } \\
= & \left(\begin{array}{cc}
{[\mathcal{W}(\lambda, \mu), \mathcal{L}(\mu)]} & 0 \\
0 & {[\mathcal{W}(\lambda, \mu), \mathcal{L}(\mu)]}
\end{array}\right) .
\end{aligned}
$$

Then comparing (3.30) and (3.31), equation (3.29) is verified.

We next address the problem of parameterizing the solutions. In fact, by introducing an elliptic (curvilinear) coordinate system $\left\{\nu_{j}^{2}\right\}$ defined by the zeros of the following function [51,60]:

$$
\mathcal{L}^{21}(\lambda)=1+\sum_{j=1}^{g} \frac{\alpha_{j} q_{j}^{2}}{\lambda^{2}-\alpha_{j}^{2}}=\frac{\mathfrak{n}(\zeta)}{\alpha(\zeta)}, \quad \mathfrak{n}(\zeta)=\prod_{j=1}^{g}\left(\zeta-\nu_{j}^{2}\right)
$$

we consider one component of the equation (3.29),

$$
\mathrm{d} \mathcal{L}^{21}(\mu) / \mathrm{d} t_{\lambda}=2\left(\mathcal{W}^{21}(\lambda, \mu) \mathcal{L}^{11}(\mu)-\mathcal{W}^{11}(\lambda, \mu) \mathcal{L}^{21}(\mu)\right),
$$

at points $\mu=\nu_{k}, 1 \leqslant k \leqslant g$. Then the Dubrovin equations for our case $[49,61]$ is obtained ${ }^{4}$,

$$
\frac{1}{2 \sqrt{R\left(\nu_{k}^{2}\right)}} \frac{\mathrm{d} \nu_{k}^{2}}{\mathrm{~d} t_{\lambda}}=-\frac{2 \delta}{\alpha(\zeta)} \frac{\mathfrak{n}(\zeta)}{\left(\zeta-\nu_{k}^{2}\right) \mathfrak{n}^{\prime}\left(\nu_{k}^{2}\right)} .
$$

Hence by using the Lagrange interpolation formula for polynomials, we get

$$
\sum_{k=1}^{g} \frac{\left(\nu_{k}^{2}\right)^{g-s}}{2 \sqrt{R\left(\nu_{k}^{2}\right)}} \frac{\mathrm{d} \nu_{k}^{2}}{\mathrm{~d} t_{\lambda}}=-\frac{2 \delta}{\alpha(\zeta)} \zeta^{g-s}, \quad(1 \leqslant s \leqslant g),
$$

which can be rewritten in the simple form

$$
\frac{\mathrm{d} \phi_{s}^{\prime}}{\mathrm{d} t_{\lambda}}=\left\{\phi_{s}^{\prime}, \mathcal{F}_{\lambda}\right\}=-\frac{2 \delta}{\alpha(\zeta)} \zeta^{g-s}, \quad(1 \leqslant s \leqslant g)
$$
${ }^{4}$ In [62] a discrete version of the Dubrovin equations was obtained associated with the finite-gap solutions of the lattice
$\mathrm{KdV}$ system. 
with the help of the quasi-Abel-Jacobi variables $\vec{\phi}^{\prime}=\left(\phi_{1}^{\prime}, \ldots, \phi_{g}^{\prime}\right)^{T}$,

$$
\phi_{s}^{\prime}=\sum_{k=1}^{g} \int_{\mathfrak{p}_{0}}^{\mathfrak{p}\left(\nu_{k}^{2}\right)} \frac{\zeta^{g-s}}{2 \sqrt{R(\zeta)}} \mathrm{d} \zeta, \quad(1 \leqslant s \leqslant g),
$$

determined by the basis of holomorphic differentials in equation (3.9). Thus, we arrive at the following proposition:

Proposition 3.3. The invariants $E_{1}, \ldots, E_{N}$ of the symplectic map $S_{\beta}$ are functionally independent throughout the phase space $\mathbb{R}^{2 N}$.

Proof. Suppose $\Sigma_{j=1}^{N} c_{j} \mathrm{~d} E_{j}=0$. Then $\Sigma_{j=1}^{N} c_{j}\left\{\phi_{s}^{\prime}, E_{j}\right\}=0, \forall s$. We shall now prove that $c_{j}=$ $0, \forall j$. Substituting the expansion (3.17) into (3.36) we get

$$
\left\{\phi_{s}^{\prime}, E_{j}\right\}=-\frac{2 \delta}{\alpha^{\prime}\left(\alpha_{j}^{2}\right)}\left(\alpha_{j}^{2}\right)^{g-s},
$$

by calculating the residues at points $\zeta=\alpha_{j}^{2}(1 \leqslant j \leqslant N)$. Then the coefficient matrix $\left(\left\{\phi_{s}^{\prime}, E_{j}\right\}\right)_{N \times N}$ is non-degenerate since its determinant is Vandermonde determinant. This completes the proof.

To summarise the results so far, the nonlinear map $S_{\beta}$ defined by (3.12) has been shown to be symplectic and integrable, possessing $N$ invariant functions $E_{1}, \ldots, E_{N}$, pairwise in involution and functionally independent on $\mathbb{R}^{2 N}$.

\section{Evolution of the solutions to Q1 equation}

In the spirit of previous papers $[17,18,26]$, we will now discuss the actual theta function solutions of the Q1 equation (1.1) arising from the integration of the symplectic map. It is the compatibility of discrete flows that is essential for constructing these solutions, and we will implement that on the relevant Baker-Akhiezer functions [39, 63]. Thus, by considering the iteration of the integrable symplectic map $S_{\beta}$ obtained in section 3, we obtain a discrete phase flow $(p(m), q(m))=S_{\beta}^{m}\left(p_{0}, q_{0}\right)$, with $\left(p_{0}, q_{0}\right) \in \mathbb{R}^{2 N}$ any initial value point. Here the number of iteration coincides with the lattice variable $m$. Then the commutative relation (3.3) along the $S_{\beta}^{m}$-flow has the form,

$$
\mathcal{L}_{m+1}(\lambda) \mathcal{D}_{m}^{(\beta)}(\lambda)=\mathcal{D}_{m}^{(\beta)}(\lambda) \mathcal{L}_{m}(\lambda),
$$

where $\mathcal{L}_{m}(\lambda)=\mathcal{L}(\lambda ; p(m), q(m))$, and $\mathcal{D}_{m}^{(\beta)}(\lambda)=\mathcal{D}^{(\beta)}\left(\lambda ; b_{m}\right)$ is the Darboux matrix given by (2.1) with discrete potentials $b_{m}, u_{m}$ satisfying

$$
\begin{aligned}
& \beta b_{m}=u_{m+1}-u_{m}, \quad \text { or } \\
& \beta b=\tilde{u}-u .
\end{aligned}
$$

We note that both the eigenvalues $\pm \mathcal{H}_{\lambda}$ given by the formula (3.5) and the spectral curve $\mathcal{R}$ defined by equation (3.8) are invariant under the $S_{\beta}^{m}$-flow, since $\mathcal{F}_{\lambda}(p(m), q(m))=\mathcal{F}_{\lambda}\left(p_{0}, q_{0}\right)$ by using equation (3.16). According to the Burchnall-Chaundy theory [35-38], we now investigate the common eigenvectors of the two matrix operators $\mathcal{L}_{m}(\lambda)$ and $\mathcal{D}_{m}^{(\beta)}(\lambda)$. Considering $\mathcal{D}_{m}^{(\beta)}(\lambda)$ as a shift operator, we suppose

$$
h(m+1, \lambda)=\mathcal{D}_{m}^{(\beta)}(\lambda) h(m, \lambda),
$$


where $h(m, \lambda)$ is the eigenvector corresponding to the eigenvalue $\lambda$. This is a linear equation, and then an alternative way of viewing $h(m, \lambda)$ is as a solution to the equation (4.3). Thus we discuss the fundamental solution matrix $\mathcal{M}(m, \lambda)$ and find

$$
\mathcal{M}(m+1, \lambda)=\mathcal{D}_{m}^{(\beta)}(\lambda) \mathcal{M}(m, \lambda), \quad \mathcal{M}(0, \lambda)=I .
$$

Then by induction, the solution can be written as a matrix product chain

$$
\mathcal{M}(m, \lambda)=\mathcal{D}_{m-1}^{(\beta)}(\lambda) \mathcal{D}_{m-2}^{(\beta)}(\lambda) \ldots \mathcal{D}_{0}^{(\beta)}(\lambda)
$$

which implies det $\mathcal{M}(m, \lambda)=\left(\lambda^{2}-\beta^{2}\right)^{m}$. Fortunately, the solution space of equation (4.3) is invariant under the action of the algebra operator $\mathcal{L}_{m}(\lambda)$. In fact, by using the commutative relation (4.1) we have

$$
(\mathcal{L} h)_{m+1}=\mathcal{L}_{m+1}\left(\mathcal{D}_{m}^{(\beta)} h_{m}\right)=\mathcal{D}_{m}^{(\beta)}(\mathcal{L} h)_{m} .
$$

Moreover, from equations (4.1) and (4.5) we obtain

$$
\mathcal{L}_{m}(\lambda) \mathcal{M}(m, \lambda)=\mathcal{M}(m, \lambda) \mathcal{L}_{0}(\lambda)
$$

which demonstrates that the evolution on the level of the Lax representation is nothing more than a (matrix) similarity transformation. Hence we consider the common eigenvectors $h_{ \pm}(m, \lambda)$ associated with the eigenvalues $\lambda$ and $\pm \mathcal{H}_{\lambda}$, which satisfy the following formulas simultaneously:

$$
\begin{aligned}
& \mathcal{L}_{m}(\lambda) h_{ \pm}(m, \lambda)= \pm \mathcal{H}_{\lambda} h_{ \pm}(m, \lambda), \\
& h_{ \pm}(m+1, \lambda)=\mathcal{D}_{m}^{(\beta)}(\lambda) h_{ \pm}(m, \lambda) .
\end{aligned}
$$

Since the rank of $\mathcal{L}_{m}(\lambda) \mp \mathcal{H}_{\lambda} I$ is 1 , the eigenvector in each case is unique (up to constant factors). Thus, the simultaneous eigenvectors $h_{ \pm}(m, \lambda)$ can be expressed as

$$
h_{ \pm}(m, \lambda)=\left(\begin{array}{l}
h_{ \pm}^{(1)}(m, \lambda) \\
h_{ \pm}^{(2)}(m, \lambda)
\end{array}\right)=\mathcal{M}(m, \lambda)\left(\begin{array}{c}
c_{\lambda}^{ \pm} \\
1
\end{array}\right)
$$

Substituting (4.9) into (4.7) and choosing $m=0$, we deduce

$$
c_{\lambda}^{ \pm}=\frac{\mathcal{L}_{0}^{11}(\lambda) \pm \mathcal{H}_{\lambda}}{\mathcal{L}_{0}^{21}(\lambda)}=\frac{-\mathcal{L}_{0}^{12}(\lambda)}{\mathcal{L}_{0}^{11}(\lambda) \mp \mathcal{H}_{\lambda}} .
$$

Referring to $[9,64,65]$, when the rank of the commuting pair, i.e., the dimension of the eigenspace of common eigenvectors, equals to 1, the associated equations of Lax type have finite-gap solutions. Here we shall investigate the common eigenvectors $h_{ \pm}(m, \lambda)$ by using the Baker-Akhiezer functions, which can be expressed by theta functions on the hyperelliptic Riemann surface corresponding to the spectral curve $\mathcal{R}$ given by (3.8). Consequently, the discrete potentials $b_{m}, u_{m}$ in equation (4.2) can be reconstructed in terms of coefficients of the asymptotic expansions of these Baker-Akhiezer functions, which leads to the theta-function solutions for the Q1 equation (1.1) [17, 18, 26].

Technically, separating out the two cases: $m=2 k-1,2 k$, by using equation (4.5) and induction, we find that the following functions are polynomials of the argument $\zeta=\lambda^{2}$ :

$$
\mathcal{M}^{21}(2 k-1, \lambda), \lambda \mathcal{M}^{22}(2 k-1, \lambda), \lambda \mathcal{M}^{21}(2 k, \lambda), \mathcal{M}^{22}(2 k, \lambda) .
$$


Furthermore, it is easy to see that $\lambda c_{\lambda}^{+}$and $\lambda c_{\lambda}^{-}$are the values of a meromorphic function on $\mathcal{R}$,

$$
\mathcal{C}(\mathfrak{p})=\frac{\zeta\left\langle\left(\zeta-A^{2}\right)^{-1} p_{0}, q_{0}\right\rangle+\delta \xi / \alpha(\zeta)}{1+\left\langle\left(\zeta-A^{2}\right)^{-1} A q_{0}, q_{0}\right\rangle},
$$

at the points $\mathfrak{p}\left(\lambda^{2}\right)$ and $(\tau \mathfrak{p})\left(\lambda^{2}\right)$, respectively. Thus, we can construct meromorphic functions $\mathfrak{h}^{(2)}(m, \mathfrak{p}),(m=2 k-1,2 k)$ on $\mathcal{R}$, i.e., Baker-Akhiezer functions, with the values at $\mathfrak{p}$ and $\tau \mathfrak{p}$ as

$$
\begin{aligned}
& \mathfrak{h}^{(2)}\left(2 k-1, \mathfrak{p}\left(\lambda^{2}\right)\right)=\lambda h_{+}^{(2)}(2 k-1, \lambda), \\
& \mathfrak{h}^{(2)}\left(2 k-1, \tau \mathfrak{p}\left(\lambda^{2}\right)\right)=\lambda h_{-}^{(2)}(2 k-1, \lambda), \\
& \mathfrak{h}^{(2)}\left(2 k, \mathfrak{p}\left(\lambda^{2}\right)\right)=h_{+}^{(2)}(2 k, \lambda), \quad \mathfrak{h}^{(2)}\left(2 k, \tau \mathfrak{p}\left(\lambda^{2}\right)\right)=h_{-}^{(2)}(2 k, \lambda),
\end{aligned}
$$

where

$$
\begin{aligned}
& \lambda h_{ \pm}^{(2)}(2 k-1, \lambda)=\mathcal{M}^{21}(2 k-1, \lambda) \lambda c_{\lambda}^{ \pm}+\lambda \mathcal{M}^{22}(2 k-1, \lambda), \\
& h_{ \pm}^{(2)}(2 k, \lambda)=\lambda^{-1} \mathcal{M}^{21}(2 k, \lambda) \lambda c_{\lambda}^{ \pm}+\mathcal{M}^{22}(2 k, \lambda) .
\end{aligned}
$$

According to the theory of Riemann surface [54-56], we now find the zeros and poles for meromorphic functions $\mathfrak{h}^{(2)}(m, \mathfrak{p}),(m=2 k-1,2 k)$, which determine the expressions in terms of theta functions.

From equations (4.9) and (4.10), we derive

$$
h_{ \pm}(m, \lambda) h_{ \pm}^{T}(m, \lambda)=\frac{1}{\mathcal{L}_{0}^{21}(\lambda)} \mathcal{M}(m, \lambda)\left[\mathcal{L}_{0}(\lambda)+\mathcal{H}_{\lambda}\right] \mathrm{i} \sigma_{2} \mathcal{M}^{T}(m, \lambda) .
$$

Then by using equations (3.32), (4.5) and (4.6), we find one entry of the above matrix equation (4.13) satisfies

$$
h_{+}^{(2)}(m, \lambda) \cdot h_{-}^{(2)}(m, \lambda)=\left(\zeta-\beta^{2}\right)^{m} \prod_{j=1}^{N} \frac{\zeta-\nu_{j}^{2}(m)}{\zeta-\nu_{j}^{2}(0)} .
$$

Thus, by equation (4.11), we have

$$
\begin{aligned}
& \mathfrak{h}^{(2)}(2 k-1, \mathfrak{p}(\zeta)) \mathfrak{h}^{(2)}(2 k-1, \tau \mathfrak{p}(\zeta))=\zeta\left(\zeta-\beta^{2}\right)^{2 k-1} \prod_{j=1}^{N} \frac{\zeta-\nu_{j}^{2}(2 k-1)}{\zeta-\nu_{j}^{2}(0)}, \\
& \mathfrak{h}^{(2)}(2 k, \mathfrak{p}(\zeta)) \mathfrak{h}^{(2)}(2 k, \tau \mathfrak{p}(\zeta))=\left(\zeta-\beta^{2}\right)^{2 k} \prod_{j=1}^{N} \frac{\zeta-\nu_{j}^{2}(2 k)}{\zeta-\nu_{j}^{2}(0)},
\end{aligned}
$$

which implies zeros and some poles, while the asymptotic behaviors in the vicinity of the infinity point on $\mathcal{R}$ will provide the remaining poles. Indeed, as $\lambda \sim \infty$, we obtain

$$
c_{\lambda}^{ \pm}= \pm \delta \lambda\left[1+O\left(\lambda^{-2}\right)\right]
$$

by using equation (4.10) and

$$
\mathcal{L}^{11}(\lambda)=O\left(\lambda^{-1}\right), \quad \mathcal{L}^{12}(\lambda)=\delta^{2} \lambda^{2}\left[1+O\left(\lambda^{-4}\right)\right],
$$




$$
\mathcal{L}^{21}(\lambda)=1+O\left(\lambda^{-2}\right), \quad \mathcal{H}_{\lambda}=\delta \lambda\left[1+O\left(\lambda^{-4}\right)\right] .
$$

Besides,

$$
\mathcal{M}(m, \lambda)=\left(\begin{array}{cc}
\lambda^{m} Z_{m}^{11}\left[1+O\left(\lambda^{-2}\right)\right] & \lambda^{m+1} Z_{m}^{12}\left[1+O\left(\lambda^{-2}\right)\right] \\
\lambda^{m-1} Z_{m}^{21}\left[1+O\left(\lambda^{-2}\right)\right] & \lambda^{m} Z_{m}^{22}\left[1+O\left(\lambda^{-2}\right)\right]
\end{array}\right)
$$

where

$$
\begin{aligned}
& Z_{m}=\left(\begin{array}{ll}
Z_{m}^{11} & Z_{m}^{12} \\
Z_{m}^{21} & Z_{m}^{22}
\end{array}\right)=\frac{1}{2 \delta}\left(\begin{array}{cc}
\delta\left(z_{m}+z_{m}^{-1}\right) & \delta^{2}\left(z_{m}-z_{m}^{-1}\right) \\
z_{m}-z_{m}^{-1} & \delta\left(z_{m}+z_{m}^{-1}\right)
\end{array}\right), \\
& z_{m}=\left(\frac{\left(b_{m-1}+\delta \beta\right) \ldots\left(b_{0}+\delta \beta\right)}{\left(b_{m-1}-\delta \beta\right) \ldots\left(b_{0}-\delta \beta\right)}\right)^{1 / 2},
\end{aligned}
$$

by equation (4.5) and induction. Substituting (4.16) and (4.17) into (4.12), we obtain the asymptotic behaviour for $\mathfrak{h}^{(2)}(m, \mathfrak{p})$ near points $\infty_{+}, \infty_{-}$:

$$
\begin{aligned}
& \begin{cases}\mathfrak{h}^{(2)}(2 k-1, \mathfrak{p})=z_{2 k-1} \zeta^{k}\left[1+O\left(\zeta^{-1}\right)\right], & \mathfrak{p} \sim \infty_{+}, \\
\mathfrak{h}^{(2)}(2 k-1, \mathfrak{p})=z_{2 k-1}^{-1} \zeta^{k}\left[1+O\left(\zeta^{-1}\right)\right], & \mathfrak{p} \sim \infty_{-} .\end{cases} \\
& \begin{cases}\mathfrak{h}^{(2)}(2 k, \mathfrak{p})=z_{2 k} \zeta^{k}\left[1+O\left(\zeta^{-1}\right)\right], & \mathfrak{p} \sim \infty_{+}, \\
\mathfrak{h}^{(2)}(2 k, \mathfrak{p})=z_{2 k}^{-1} \zeta^{k}\left[1+O\left(\zeta^{-1}\right)\right], & \mathfrak{p} \sim \infty_{-} .\end{cases}
\end{aligned}
$$

This leads to the following conclusion on the analytic behaviours of the Baker-Akhiezer functions.

Proposition 4.1. The Baker-Akhiezer functions $\mathfrak{h}^{(2)}(2 k-1, \mathfrak{p}), \mathfrak{h}^{(2)}(2 k, \mathfrak{p})$ on $\mathcal{R}$ have the following divisors, respectively [54-56]:

$$
\begin{aligned}
\operatorname{Div}\left(\mathfrak{h}^{(2)}(2 k-1, \mathfrak{p})\right)= & \sum_{j=1}^{g}\left(\mathfrak{p}\left(\nu_{j}^{2}(2 k-1)\right)-\mathfrak{p}\left(\nu_{j}^{2}(0)\right)\right) \\
& +\{\mathfrak{o}\}+(2 k-1) \mathfrak{p}\left(\beta^{2}\right)-k \infty_{+}-k \infty_{-}, \\
\operatorname{Div}\left(\mathfrak{h}^{(2)}(2 k, \mathfrak{p})\right)= & \sum_{j=1}^{g}\left(\mathfrak{p}\left(\nu_{j}^{2}(2 k)\right)-\mathfrak{p}\left(\nu_{j}^{2}(0)\right)\right) \\
& +2 k \mathfrak{p}\left(\beta^{2}\right)-k \infty_{+}-k \infty_{-} .
\end{aligned}
$$

Let us now introduce the Abel-Jacobi variable $\vec{\phi}(m)=\mathscr{A}\left(\sum_{k=1}^{g} \mathfrak{p}\left(\nu_{k}^{2}(m)\right)\right)$ on $J(\mathcal{R})=\mathbb{C}^{g} / \mathscr{T}$, with the help of the Able map $\mathscr{A}$ given in (3.11). Resorting to the dipole technique developed by [66], equation (4.22) implies

$$
\begin{aligned}
& \vec{\phi}(2 k-1) \equiv \vec{\phi}(0)+k \vec{\Omega}_{\beta}^{+}+(k-1) \vec{\Omega}_{\beta}^{-}+\vec{\Omega}_{0}^{-}, \quad(\bmod \mathscr{T}), \\
& \vec{\phi}(2 k) \equiv \vec{\phi}(0)+k \vec{\Omega}_{\beta}^{+}+k \vec{\Omega}_{\beta}^{-}, \quad(\bmod \mathscr{T}),
\end{aligned}
$$

which can be rewritten as

$$
\vec{\phi}(m) \equiv \vec{\phi}(0)+\frac{m+\Delta_{m}}{2} \vec{\Omega}_{\beta}^{+}+\frac{m-\Delta_{m}}{2} \vec{\Omega}_{\beta}^{-}+\Delta_{m} \vec{\Omega}_{0}^{-}, \quad(\bmod \mathscr{T}),
$$


where $\vec{\Omega}_{\beta}^{+}=\int_{\mathfrak{p}\left(\beta^{2}\right)}^{\infty+} \vec{\omega}, \vec{\Omega}_{\beta}^{-}=\int_{\mathfrak{p}\left(\beta^{2}\right)}^{\infty-} \vec{\omega}$ and $\vec{\Omega}_{0}^{-}=\int_{\mathcal{O}}^{\infty-} \vec{\omega}$. Moreover, $\Delta_{j}$ is equal to 0 and 1 for even and odd $j$ respectively.

A usual argument leads to the expressions for the Baker-Akhiezer functions as $[17,54-56]$

$$
\begin{aligned}
\mathfrak{h}^{(2)}(2 k-1, \mathfrak{p})= & C_{2 k-1} \cdot \frac{\theta[-\mathscr{A}(\mathfrak{p})+\vec{\phi}(2 k-1)+\vec{K}]}{\theta[-\mathscr{A}(\mathfrak{p})+\vec{\phi}(0)+\vec{K}]} \\
& \times e^{\int_{\mathfrak{p}_{0}}^{\mathfrak{p} k \omega\left[\mathfrak{p}\left(\beta^{2}\right), \infty_{+}\right]+(k-1) \omega\left[\mathfrak{p}\left(\beta^{2}\right), \infty_{-}\right]+\omega\left[\mathfrak{o}, \infty_{-}\right]},} \\
\mathfrak{h}^{(2)}(2 k, \mathfrak{p})=C_{2 k} & \cdot \frac{\theta[-\mathscr{A}(\mathfrak{p})+\vec{\phi}(2 k)+\vec{K}]}{\theta[-\mathscr{A}(\mathfrak{p})+\vec{\phi}(0)+\vec{K}]} e^{\int_{\mathfrak{p}_{0}}^{\mathfrak{p}} k \omega\left[\mathfrak{p}\left(\beta^{2}\right), \infty_{+}\right]+k \omega\left[\mathfrak{p}\left(\beta^{2}\right), \infty_{-}\right]},
\end{aligned}
$$

with $C_{2 k-1}, C_{2 k}$ the constants; $\vec{K}$ the Riemann constant; $\omega[p, q]$ the dipole, a meromorphical differential having only simple poles at $p, q$, with residues $+1,-1$, respectively.

From asymptotic behaviours (4.20) and (4.21), we obtain

$$
\begin{aligned}
z_{2 k-1} & =C_{2 k-1} \cdot \frac{\theta\left[-\mathscr{A}\left(\infty_{+}\right)+\vec{\phi}(2 k-1)+\vec{K}\right]}{\theta\left[-\mathscr{A}\left(\infty_{+}\right)+\vec{\phi}(0)+\vec{K}\right]} \times e^{\int_{\mathfrak{p}_{0}}^{\infty_{+}}(k-1) \omega\left[\mathfrak{p}\left(\beta^{2}\right), \infty_{-}\right]+\omega\left[0, \infty_{-}\right]} \cdot\left(r_{\beta}^{+}\right)^{k}, \\
z_{2 k-1}^{-1} & =C_{2 k-1} \cdot \frac{\theta\left[-\mathscr{A}\left(\infty_{-}\right)+\vec{\phi}(2 k-1)+\vec{K}\right]}{\theta\left[-\mathscr{A}\left(\infty_{-}\right)+\vec{\phi}(0)+\vec{K}\right]} e^{\int_{\mathfrak{p}_{0}}^{\infty_{-}}{ }^{k \omega\left[\mathfrak{p}\left(\beta^{2}\right), \infty_{+}\right]} \cdot\left(r_{\beta}^{-}\right)^{k-1} \cdot r_{0}^{-},} \\
z_{2 k} & =C_{2 k} \cdot \frac{\theta\left[-\mathscr{A}\left(\infty_{+}\right)+\vec{\phi}(2 k)+\vec{K}\right]}{\theta\left[-\mathscr{A}\left(\infty_{+}\right)+\vec{\phi}(0)+\vec{K}\right]} e^{\int_{\mathfrak{p}_{0}}^{\infty} k \omega\left[\mathfrak{p}\left(\beta^{2}\right), \infty_{-}\right]} \cdot\left(r_{\beta}^{+}\right)^{k}, \\
z_{2 k}^{-1} & =C_{2 k} \cdot \frac{\theta\left[-\mathscr{A}\left(\infty_{-}\right)+\vec{\phi}(2 k)+\vec{K}\right]}{\theta\left[-\mathscr{A}\left(\infty_{-}\right)+\vec{\phi}(0)+\vec{K}\right]} e^{\int_{\mathfrak{p}_{0}}^{\infty_{-}} k \omega\left[\mathfrak{p}\left(\beta^{2}\right), \infty_{+}\right]} \cdot\left(r_{\beta}^{-}\right)^{k},
\end{aligned}
$$

where

$$
\begin{aligned}
& r_{\beta}^{+}=\lim _{\mathfrak{p} \rightarrow \infty^{+}} \frac{1}{\zeta(\mathfrak{p})} e^{\int_{\mathfrak{p}_{0}}^{\mathfrak{p}} \omega\left[\mathfrak{p}\left(\beta^{2}\right), \infty_{+}\right]}, \quad r_{\beta}^{-}=\lim _{\mathfrak{p} \rightarrow \infty^{-}} \frac{1}{\zeta(\mathfrak{p})} e^{\int_{\mathfrak{p}_{0}}^{\mathfrak{p} \omega\left[\mathfrak{p}\left(\beta^{2}\right), \infty_{-}\right]},} \\
& r_{0}^{-}=\lim _{\mathfrak{p} \rightarrow \infty^{-}} \frac{1}{\zeta(\mathfrak{p})} e^{\int_{\mathfrak{p}_{0}}^{\mathfrak{p} \omega\left[\mathfrak{o}, \infty_{-}\right]}} .
\end{aligned}
$$

Hence

$$
\begin{aligned}
z_{2 k-1}^{2}= & \frac{\theta\left[-\mathscr{A}\left(\infty_{+}\right)+\vec{\phi}(2 k-1)+\vec{K}\right]}{\theta\left[-\mathscr{A}\left(\infty_{-}\right)+\vec{\phi}(2 k-1)+\vec{K}\right]} \cdot \frac{\theta\left[-\mathscr{A}\left(\infty_{-}\right)+\vec{\phi}(0)+\vec{K}\right]}{\theta\left[-\mathscr{A}\left(\infty_{+}\right)+\vec{\phi}(0)+\vec{K}\right]} \\
& \cdot\left(\frac{r_{\beta}^{+}}{r_{\beta}^{-}}\right)^{k-1} \cdot \frac{r_{\beta}^{+}}{r_{0}^{-}} \cdot e^{\int_{\mathfrak{p}_{0}}^{\infty}(k-1) \omega\left[\mathfrak{p}\left(\beta^{2}\right), \infty_{-}\right]+\omega\left[0, \infty_{-}\right]-\int_{\mathfrak{p}_{0}}^{\infty} k \omega\left[\mathfrak{p}\left(\beta^{2}\right), \infty_{+}\right]}, \\
z_{2 k}^{2}= & \frac{\theta\left[-\mathscr{A}\left(\infty_{+}\right)+\vec{\phi}(2 k)+\vec{K}\right]}{\theta\left[-\mathscr{A}\left(\infty_{-}\right)+\vec{\phi}(2 k)+\vec{K}\right]} \cdot \frac{\theta\left[-\mathscr{A}\left(\infty_{-}\right)+\vec{\phi}(0)+\vec{K}\right]}{\theta\left[-\mathscr{A}\left(\infty_{+}\right)+\vec{\phi}(0)+\vec{K}\right]} \\
& \cdot\left(\frac{r_{\beta}^{+}}{r_{\beta}^{-}}\right)^{k} \cdot e^{\int_{\mathfrak{p}_{0}}^{\infty} k \omega\left[\mathfrak{p}\left(\beta^{2}\right), \infty_{-}\right]-\int_{\mathfrak{p}_{0}}^{\infty} k \omega\left[\mathfrak{p}\left(\beta^{2}\right), \infty_{+}\right]} .
\end{aligned}
$$


Then by using equation (4.19), we have

$$
\begin{aligned}
\frac{b_{2 k-1}+\delta \beta}{b_{2 k-1}-\delta \beta}=\frac{z_{2 k}^{2}}{z_{2 k-1}^{2}}= & \frac{\theta\left[-\mathscr{A}\left(\infty_{+}\right)+\vec{\phi}(2 k)+\vec{K}\right]}{\theta\left[-\mathscr{A}\left(\infty_{-}\right)+\vec{\phi}(2 k)+\vec{K}\right]} \cdot \frac{\theta\left[-\mathscr{A}\left(\infty_{-}\right)+\vec{\phi}(2 k-1)+\vec{K}\right]}{\theta\left[-\mathscr{A}\left(\infty_{+}\right)+\vec{\phi}(2 k-1)+\vec{K}\right]} \\
& \cdot \frac{r_{0}^{-}}{r_{\beta}^{-}} \cdot e^{\int_{\mathfrak{p}_{0}}^{\infty_{+}} \omega\left[\mathfrak{p}\left(\beta^{2}\right), \infty_{-}\right]-\omega\left[\mathfrak{o}_{-} \infty_{-}\right]}, \\
\frac{b_{2 k}+\delta \beta}{b_{2 k}-\delta \beta}=\frac{z_{2 k+1}^{2}}{z_{2 k}^{2}}= & \frac{\theta\left[-\mathscr{A}\left(\infty_{+}\right)+\vec{\phi}(2 k+1)+\vec{K}\right]}{\theta\left[-\mathscr{A}\left(\infty_{-}\right)+\vec{\phi}(2 k+1)+\vec{K}\right]} \cdot \frac{\theta\left[-\mathscr{A}\left(\infty_{-}\right)+\vec{\phi}(2 k)+\vec{K}\right]}{\theta\left[-\mathscr{A}\left(\infty_{+}\right)+\vec{\phi}(2 k)+\vec{K}\right]} \\
& \cdot \frac{r_{\beta}^{+}}{r_{0}^{-}} \cdot e^{\int_{\mathfrak{p}_{0}}^{\infty_{+}} \omega\left[\boldsymbol{o}_{0} \infty_{-}\right]-\int_{\mathfrak{p}_{0}}^{\infty_{-}} \omega\left[\mathfrak{p}\left(\beta^{2}\right), \infty_{+}\right]},
\end{aligned}
$$

which can be put in a unified form by introducing the function

$$
\begin{aligned}
\Upsilon_{m}=\frac{b_{m}+\delta \beta}{b_{m}-\delta \beta}= & \frac{\theta\left[-\mathscr{A}\left(\infty_{+}\right)+\vec{\phi}(m+1)+\vec{K}\right]}{\theta\left[-\mathscr{A}\left(\infty_{-}\right)+\vec{\phi}(m+1)+\vec{K}\right]} \cdot \frac{\theta\left[-\mathscr{A}\left(\infty_{-}\right)+\vec{\phi}(m)+\vec{K}\right]}{\theta\left[-\mathscr{A}\left(\infty_{+}\right)+\vec{\phi}(m)+\vec{K}\right]} \\
& \cdot \frac{\left(r_{\beta}^{+}\right)^{\Delta_{m+1}}}{\left(r_{\beta}^{-}\right)^{\Delta_{m}}} \cdot\left(r_{0}^{-}\right)^{(-1)^{\Delta_{m+1}}} \\
& \cdot e^{\int_{\mathfrak{p}_{0}}^{\infty_{+}} \Delta_{m} \omega\left[\mathfrak{p}\left(\beta^{2}\right), \infty_{-}\right]+(-1)^{\Delta_{m}} \omega\left[\mathfrak{o}, \infty_{-}\right]-\Delta_{m+1} \int_{\mathfrak{p}_{0}}^{\infty_{-}} \omega\left[\mathfrak{p}\left(\beta^{2}\right), \infty_{+}\right]},
\end{aligned}
$$

from which we have by inverting the definition

$$
b_{m}=\frac{\delta \beta\left(\Upsilon_{m}+1\right)}{\Upsilon_{m}-1}
$$

Substituting equation (4.2) into (4.33) we arrive at a recursive relation for the potential $u_{m}$, in terms of theta functions,

$$
u_{m+1}-u_{m}=\frac{\delta \beta^{2}\left(\Upsilon_{m}+1\right)}{\Upsilon_{m}-1} .
$$

In order to get the algebro-geometric solutions to equation (1.1), we introduce two distinct and non-zero lattice parameters $\beta_{1}, \beta_{2}$. According to the results in section 3 , we obtain two commuting integrable maps $S_{\beta_{1}}, S_{\beta_{2}}$ since they share the same invariants $E_{1}, \ldots, E_{N}$ [22-26]. Their iteration give rise to commuting discrete flows $S_{\beta_{1}}^{m}$ and $S_{\beta_{2}}^{n}$. Consequently the following function is well-defined on the $\mathbb{Z}^{2}$ lattice:

$$
\begin{aligned}
(p(m, n), q(m, n)) & =S_{\beta_{1}}^{m} S_{\beta_{2}}^{n}\left(p_{0}, q_{0}\right)=S_{\beta_{1}}^{m}(p(0, n), q(0, n)) \\
& =S_{\beta_{2}}^{n} S_{\beta_{1}}^{m}\left(p_{0}, q_{0}\right)=S_{\beta_{2}}^{n}(p(m, 0), q(m, 0)) .
\end{aligned}
$$

Furthermore, by equations (3.1) in the two special cases and (4.2), the $j$ th component satisfies

$$
\begin{aligned}
& \left(\begin{array}{l}
\tilde{p}_{j} \\
\tilde{q}_{j}
\end{array}\right)=\left(\alpha_{j}^{2}-\beta_{1}^{2}\right)^{-1 / 2} \mathcal{D}^{\left(\beta_{1}\right)}\left(\alpha_{j} ; b_{1}\right)\left(\begin{array}{c}
p_{j} \\
q_{j}
\end{array}\right), \quad b_{1}=\frac{\tilde{u}-u}{\beta_{1}} \\
& \left(\begin{array}{l}
\bar{p}_{j} \\
\bar{q}_{j}
\end{array}\right)=\left(\alpha_{j}^{2}-\beta_{2}^{2}\right)^{-1 / 2} \mathcal{D}^{\left(\beta_{2}\right)}\left(\alpha_{j} ; b_{2}\right)\left(\begin{array}{c}
p_{j} \\
q_{j}
\end{array}\right), \quad b_{2}=\frac{\bar{u}-u}{\beta_{2}},
\end{aligned}
$$


which are compatible on account of the commutative relation between the maps $S_{\beta_{1}}$ and $S_{\beta_{2}}$. Thus $\bar{D}^{\left(\beta_{1}\right)} D^{\left(\beta_{2}\right)}=\tilde{D}^{\left(\beta_{2}\right)} D^{\left(\beta_{1}\right)}$. Then by using equation $(2.3)$, the evolution of the recursive relation (4.34) along the flows $S_{\beta_{1}}^{m}$ and $S_{\beta_{2}}^{n}$ leads to the solutions for Q1 lattice equation (1.1) in the form as expressed in the following.

Proposition 4.2. The $Q 1$ equation (1.1) has special solutions $u=u_{m, n}$ satisfying

$$
u_{m+1, n}-u_{m, n}=\frac{\delta \beta_{1}^{2}\left(\Upsilon_{m, n}+1\right)}{\Upsilon_{m, n}-1}
$$

where

$$
\begin{aligned}
\Upsilon_{m, n}= & \frac{\theta\left[-\mathscr{A}\left(\infty_{+}\right)+\vec{\phi}(m+1, n)+\vec{K}\right]}{\theta\left[-\mathscr{A}\left(\infty_{-}\right)+\vec{\phi}(m+1, n)+\vec{K}\right]} \cdot \frac{\theta\left[-\mathscr{A}\left(\infty_{-}\right)+\vec{\phi}(m, n)+\vec{K}\right]}{\theta\left[-\mathscr{A}\left(\infty_{+}\right)+\vec{\phi}(m, n)+\vec{K}\right]} \\
& \cdot \frac{\left(r_{\beta_{1}}^{+}\right)^{\Delta_{m+1}}}{\left(r_{\beta_{1}}^{-}\right)^{\Delta_{m}}} \cdot\left(r_{0}^{-}\right)^{(-1)^{\Delta_{m+1}}} \\
& \cdot e^{\int_{\mathfrak{p}_{0}}^{\infty_{+}} \Delta_{m} \omega\left[\mathfrak{p}\left(\beta_{1}^{2}\right), \infty_{-}\right]+(-1)^{\Delta_{m} \omega\left[\mathfrak{o}, \infty_{-}\right]-\Delta_{m+1} \int_{\mathfrak{p}_{0}}^{\infty_{-}} \omega\left[\mathfrak{p}\left(\beta_{1}^{2}\right), \infty_{+}\right]},} \\
\vec{\phi}(m, n) \equiv & \vec{\phi}(0,0)+\frac{m+\Delta_{m}}{2} \vec{\Omega}_{\beta_{1}}^{+}+\frac{m-\Delta_{m}}{2} \vec{\Omega}_{\beta_{1}}^{-} \\
& +\frac{n+\Delta_{n}}{2} \vec{\Omega}_{\beta_{2}}^{+}+\frac{n-\Delta_{n}}{2} \vec{\Omega}_{\beta_{2}}^{-}+\left(\Delta_{m}+\Delta_{n}\right) \vec{\Omega}_{0}^{-}, \quad(\bmod \mathscr{T}),
\end{aligned}
$$

and $\vec{\Omega}_{\beta_{j}}^{+}=\int_{\mathfrak{p}\left(\beta_{j}^{2}\right)}^{\infty+} \vec{\omega}, \vec{\Omega}_{\beta_{j}}^{-}=\int_{\mathfrak{p}\left(\beta_{j}^{2}\right)}^{\infty} \vec{\omega}, \quad j=1,2$.

Besides,

$$
u_{m, n+1}-u_{m, n}=\frac{\delta \beta_{2}^{2}\left(\Theta_{m, n}+1\right)}{\Theta_{m, n}-1},
$$

where

$$
\begin{aligned}
\Theta_{m, n}= & \frac{\theta\left[-\mathscr{A}\left(\infty_{+}\right)+\vec{\phi}(m, n+1)+\vec{K}\right]}{\theta\left[-\mathscr{A}\left(\infty_{-}\right)+\vec{\phi}(m, n+1)+\vec{K}\right]} \cdot \frac{\theta\left[-\mathscr{A}\left(\infty_{-}\right)+\vec{\phi}(m, n)+\vec{K}\right]}{\theta\left[-\mathscr{A}\left(\infty_{+}\right)+\vec{\phi}(m, n)+\vec{K}\right]} \\
& \cdot \frac{\left(r_{\beta_{2}}^{+}\right)^{\Delta_{n+1}}}{\left(r_{\beta_{2}}^{-}\right)^{\Delta_{n}}} \cdot\left(r_{0}^{-}\right)^{(-1)^{\Delta_{n+1}}} \\
& \cdot e^{\int_{\mathfrak{p}_{0}}^{\infty_{+}} \Delta_{n} \omega\left[\mathfrak{p}\left(\beta_{2}^{2}\right), \infty_{-}\right]+(-1)^{\Delta_{n}} \omega\left[\mathfrak{o}, \infty_{-}\right]-\Delta_{n+1} \int_{\mathfrak{p}_{0}}^{\infty_{-}} \omega\left[\mathfrak{p}\left(\beta_{2}^{2}\right), \infty_{+}\right]} .
\end{aligned}
$$

This proposition forms the main and final result of the paper. We remark that based on (4.38) and (4.40) the solutions to Q1 equation (1.1), in terms of theta functions, should be integrated in order to find $u=u_{m, n}$ in the form

$$
\begin{aligned}
u & =u_{0, n}+\sum_{j=1}^{m}\left(u_{j, n}-u_{j-1, n}\right) \\
& =u_{m, 0}+\sum_{j=1}^{n}\left(u_{m, j}-u_{m, j-1}\right) .
\end{aligned}
$$

However, it is not clear yet that the sum (4.42) can be explicitly computed in closed form. 


\section{Conclusion}

In this paper we constructed algebro-geometric solutions of the Q1 equation (1.1) in a novel way different from earlier approaches applied in e.g. $\operatorname{KdV}$ type systems $[17,18,26]$ where the continuous spectral problems associated with the integrable Hamiltonian systems in the Liouville sense are essential. Here only the lattice equation is the starting point which gives rise to the purely discrete Lax pair by means of the multidimensional consistency. From the latter we deduce the compatibility relations, as well as the relevant spectral curve and the associated hyperelliptic Riemann surface. Moreover, the integrability for the symplectic maps are studied with the help of the $r$-matrix. An outstanding new feature in the present approach is the revelation that the discrete systems themselves without the continuous integrability provides enough information for calculating the exact analytic solutions by the finite-gap technique. Thus, in a sense it is justified to consider the discrete integrability as the more fundamental aspect, which can subsequently be used to investigate the associated continuous integrable systems.

We note, however, that the solutions are obtained in a 'derived' form, which to obtain the solution, should still be integrated (in the discrete sense), to get the algebro-geometric solutions for Q1 lattice equation in explicit form. Whether or not those forms can be explicitly integrated remains an open problem, but there are precedents in the case of soliton solutions where that can be done (see [67]). However, the latter, which has never been achieved in the finite-gap case, is a matter for future investigation beyond the scope of the present paper.

We finish by expressing our confidence that the techniques used in the present paper for the Q1 equation can be readily extended to the remaining equations in the ABS list which are beyond the KdV type class, and notably to the problem of constructing algebro-geometric solutions of the Q4 equation which figures at the top of the list.

\section{Acknowledgments}

This work is supported by National Natural Science Foundation of China (Grant Nos. 11426206; 11501521), State Scholarship Found of China (CSC No. 201907045035), and Graduate Student Education Research Foundation of Zhengzhou University (Grant No. YJSXWKC201913). We would like to express many thanks to Prof. Da-jun Zhang for helpful discussions.

\section{ORCID iDs}

Frank W Nijhoff (D) https://orcid.org/0000-0002-1520-0502

\section{References}

[1] Hietarinta J, Joshi N and Nijhoff F W 2016 Discrete Systems and Integrability (Cambridge: Cambridge University Press)

[2] Babelon O, Bernard D and Talon M 2003 Introduction to Classical Integrable Systems (Cambridge: Cambridge University Press)

[3] Bobenko A I and Yu S 2009 Discrete Differential Geometry (Berlin: Springer)

[4] Adler V E, Bobenko A I and Suris Y B 2003 Classification of integrable equations on quad-graphs. The consistency approach Commun. Math. Phys. 233 513-43

[5] Nijhoff F and Capel H 1995 The discrete Korteweg-de Vries equation Acta Appl. Math. 39 133-58 
[6] Nijhoff F, Atkinson J and Hietarinta J 2009 Soliton solutions for ABS lattice equations: I. Cauchy matrix approach J. Phys. A: Math. Theor. 42404005

[7] Nijhoff F W and Atkinson J 2010 Elliptic $N$-soliton solutions of ABS lattice equations Int. Math. Res. Not. $20103837-95$

[8] Atkinson J and Nijhoff F 2010 A constructive approach to the soliton solutions of integrable quadrilateral lattice equations Commun. Math. Phys. 299 283-304

[9] Krichever I M and Novikov S P 1980 Holomorphic bundles over algebraic curves and nonlinear equations Russ. Math. Surv. 35 53-79

[10] Weiss J 1983 The Painlevé property for partial differential equations. II: Bäcklund transformations, Lax pairs, and the Schwarzian derivative J. Math. Phys. 24 1405-13

[11] Bobenko A and Pinkall U 1996 Discrete surfaces with constant negative Gaussian curvature and the Hirota equation J. Differ. Geom. 43 527-611

[12] Hertrich-Jeromin U, McIntosh I, Norman P and Pedit F 2001 Periodic discrete conformal maps $J$. Reine Angew. Math. 534 129-53

[13] Xu X, Jiang M and Nijhoff F W 2020 Integrable symplectic maps associated with discrete Korteweg-de Vries-type equations Stud. Appl. Math. 146 233-78

[14] Xu X, Cao C and Zhang G 2020 Finite genus solutions to the lattice Schwarzian Korteweg-de Vries equation J. Nonlinear Math. Phys. 27 633-46

[15] Cao C W 1990 Nonlinearization of the Lax system for AKNS hierarchy Sci. China Ser. A 33 528-36

[16] Cao C W 1991 A classical integrable system and the involutive representation of solutions of the KdV equations Acta Math. Sin. 7 216-23

[17] Cao C and Xu X 2012 A finite genus solution of the H1 model J. Phys. A: Math. Theor. 45055213

[18] Cao C and Zhang G 2012 A finite genus solution of the Hirota equation via integrable symplectic maps J. Phys. A: Math. Theor. 45095203

[19] Shi Y and Zhang D J 2011 Rational solutions of the H3 and Q1 models in the ABS lattice list Symmetry, Integrability Geometry Methods Appl. 7 1-11

[20] Matveev V B 200830 years of finite-gap integration theory Phil. Trans. R. Soc. A 366 837-75

[21] Quispel G R W, Roberts J A G and Thompson C J 1988 Integrable mappings and soliton equations Phys. Lett. A 126 419-21

[22] Veselov A P 1991 Integrable maps Russ. Math. Surv. 46 3-45

[23] Veselov A P 1991 What Is an Integrable Mapping? What Is Integrability? (Berlin: Springer)

[24] Bruschi M, Ragnisco O, Santini P M and Gui-Zhang T 1991 Integrable symplectic maps Physica D 49 273-94

[25] Suris Y B 2003 The Problem of Integrable Discretization: Hamiltonian Approach (Basel: Birkhäuser)

[26] Cao C and Zhang G 2012 Integrable symplectic maps associated with the ZS-AKNS spectral problem J. Phys. A: Math. Theor. $\mathbf{4 5} 265201$

[27] Nijhoff F W 2002 Lax pair for the Adler (lattice Krichever-Novikov) system Phys. Lett. A 297 $49-58$

[28] Adler V E and Suris Y 2004 Q4: integrable master equation related to an elliptic curve Int. Math. Res. Not. $20042523-53$

[29] Adler V 1998 Bäcklund transformation for the Krichever-Novikov equation Int. Math. Res. Not. $19981-4$

[30] Levi D and Benguria R 1980 Bäcklund transformations and nonlinear differential difference equations Proc. Natl Acad. Sci. 77 5025-7

[31] Levi D 1981 Nonlinear differential difference equations as Bäcklund transformations J. Phys. A: Math. Gen. 14 1083-98

[32] Belokolos E D, Bobenko A I, Enolskij V Z, Its A R and Matveev V B 1994 Algebro-Geometric Approach to Nonlinear Integrable Equations (Berlin: Spinger)

[33] Bobenko A I and Bordag L A 1987 Qualitative analysis of finite-zonal solutions of the KdV equation with the help of an automorphic approach Zap. Nauchn. Sem. LOMI 165 31-41

[34] Bobenko A I and Bordag L A 1989 Periodic multiphase solutions of the KP equation J. Phys. A: Math. Gen. 22 1259-74

[35] Burchnall J L and Chaundy T W 1923 Commutative ordinary differential operators Proc. Lond. Math. Soc. s2-21 420-40

[36] Burchnall J L and Chaundy T W 1928 Commutative ordinary differential operators Proc. R. Soc. A $118557-83$ 
[37] Naiman P B 1962 On the theory of periodic and limit-periodic Jacobian matrices Dokl. Akad. Nauk SSSR 143 277-9

[38] Glazman I M 1963 Direct Methods of Qualitative Spectral Analysis of Singular Differential Operators (Moscow: Fizmatgiz)

[39] Akhiezer N I 1961 Continuous analogues of orthogonal polynomials on a system of intervals Dokl. Akad. Nauk SSSR $141263-6$

[40] Its A R and Matveev V B 1975 Schrödinger operators with the finite-band spectrum and the $N$ soliton solutions of the Korteweg-de Vries equation Theor. Math. Phys. 23 343-55

[41] Krichever I M 1977 Methods of algebraic geometry in the theory of non-linear equations Russ. Math. Surv. 32 185-213

[42] Akhiezer N I 1960 Orthogonal polynomials on several intervals Dokl. Akad. Nauk SSSR 134 9-12

[43] Kac M and van Moerbeke P 1975 On some periodic Toda lattices Proc. Natl Acad. Sci. 72 1627-9

[44] Dubrovin B A, Matveev V B and Novikov S P 1976 Nonlinear equations of Korteweg-de Vries type, finite-zone linear operators and Abelian varieties Russ. Math. Surv. 31 56-134

[45] Date E and Tanaka S 1976 Periodic multi-soliton solutions of Korteweg-de Vries equation and Toda lattice Prog. Theor. Phys. Suppl. 59 107-25

[46] Flaschka H and McLaughlin D W 1976 Canonically conjugate variables for the Korteweg-de Vries Equation and Toda lattice with periodic boundary conditions Prog. Theor. Phys. 55 438-56

[47] Mumford D and van Moerbeke P 1979 Spectrum of difference operators and algebraic curve Acta Math. 143 93-154

[48] Krichever I M and Novikov S P 2003 Two-dimensional Toda lattice, commuting difference operators and holomorphic bundles Russ. Math. Surv. 58 50-88

[49] Gesztesy F, Holden H, Michor J and Teschl G 2009 Soliton Equations and There Algebro-Geometric Solutions $((1+1)$-Dimensional Discrete Models vol 2) (Cambridge: Cambridge University Press)

[50] Geng X, Wei J and Zeng X 2019 Algebro-geometric integration of the modified Belov-Chaltikian lattice hierarchy Theor. Math. Phys. 199 675-94

[51] Arnold V I 1978 Mathematical Methods in Classical Mechanics (Berlin: Springer)

[52] Goldstein H 1980 Classical Mechanics (Reading, MA: Addison-Wesley)

[53] Jordan T F 2004 Steppingstones in Hamiltonian dynamics Am. J. Phys. 72 1095-9

[54] Griffiths P and Harris J 1978 Principles of Algebraic Geometry (New York: Wiley)

[55] Mumford D 1983 Tata Lectures on Theta I (Boston, MA: Birkhäuser)

[56] Farkas H M and Kra I 1992 Riemann Surfaces (New York: Springer)

[57] Faddeev L D and Takhtajan L A 1987 Hamiltonian Methods in the Theory of Solitons (Berlin: Springer)

[58] Gerdjikov V S, Vilasi G and Yanovski A B 2008 Integrable Hamiltonian Hierarchies (Berlin: Springer)

[59] Cao C, Geng X and Wu Y 1999 From the special $2+1$ Toda lattice to the Kadomtsev-Petviashvil equation J. Phys. A: Math. Gen. 32 8059-78

[60] Lamé G 1859 Leçons sur les Coordonnées Curvilignes et leurs Diverses Applications (Paris: MalletBachelier)

[61] Dubrovin B A 1975 Periodic problems for the Korteweg-de Vries equation in the class of finite band potentials Funct. Anal. Appl. $9215-23$

[62] Nijhoff F W 2000 Discrete Dubrovin equations and separation of variables for discrete systems Chaos Solitons Fractals 11 19-28

[63] Baker H F 1928 Note on the foregoing paper, 'commutative ordinary differential operators', by J L Burchnall and J W Chaundy Proc. R. Soc. A 118 584-93

[64] Krichever I M 1976 An algebraic-geometrical construction of the Zakharov-Shabat equations and their periodic solutions Dokl. Akad. Nauk SSSR 227 291-4

[65] Krichever I M 1977 Integration of nonlinear equations by methods of algebraic geometry Funct. Anal. Appl. 11 12-26

[66] Toda M 1981 Theory of Nonlinear Lattices (Berlin: Springer)

[67] Atkinson J, Hietarinta J and Nijhoff F 2008 Soliton solutions for Q3 J. Phys. A: Math. Theor. 41 142001 